Document downloaded from:

http://hdl.handle.net/10251/155399

This paper must be cited as:

Casares-Giner, V.; Martínez Bauset, J.; Portillo, C. (2019). Performance evaluation of framed slotted ALOHA with reservation packets and succesive interference cancelation for M2M networks. Computer Networks. 155:15-30.

https://doi.org/10.1016/j.comnet.2019.02.021

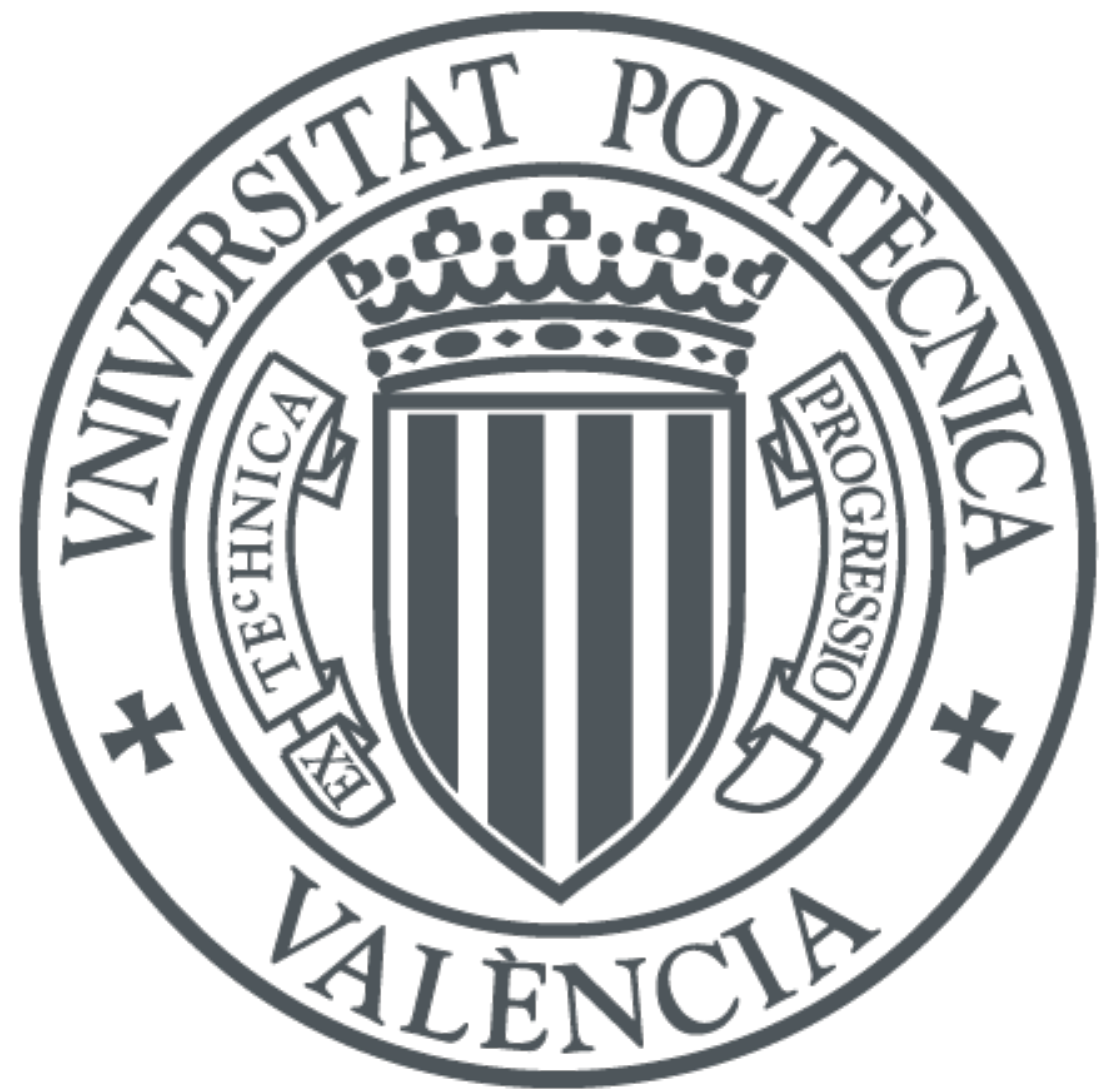

The final publication is available at

https://doi.org/10.1016/j.comnet.2019.02.021

Copyright Elsevier

Additional Information 


\title{
Performance Evaluation of Framed Slotted ALOHA with Reservation Packets and Succesive Interference Cancelation for M2M Networks
}

\author{
Vicente Casares-Giner, Jorge Martinez-Bauset, Canek Portillo \\ Instituto ITACA, Universitat Politècnica de València, Spain
}

\begin{abstract}
Random access protocols like ALOHA have been considered for machine-to-machine (M2M) communication in future networks for their simplicity of operation. This paper evaluates the performance of a Frame Slotted-ALOHA protocol that uses reservation and data packets (FSA-RDP), in a scenario where a controller collects data packets transmitted by a finite number of M2M devices. In FSA-RDP, frames of variable duration are divided in two parts, the reservation and data subframes. During the reservation subframe, active devices send short reservation packets to the controller. The controller assigns reserved slots in the data subframe to those devices that succeeded with the reservation. At devices, the FIFO service discipline and two queue management schemes, tail drop and push-out, have been considered. When the queue size is of one packet, we develop a discrete-time Markov chain to evaluate the protocol performance, including the cumulative distribution function of the delay of data packets that are successfully transmitted. Analytical results are validated by extensive simulations. The simulation model is also used to evaluate the system performance when larger queues are used. In addition, we study the impact that implementing Successive Interference Cancellation (SIC) at the controller has on the system performance. We also evaluate the performance of implementing SIC at the controller together with Irregular Repetition Slotted ALOHA (IRSA) to send the reservation packets. Numerical results show that the protocol efficiency of FSA-RDP is between one and two orders of magnitude larger than the efficiency of conventional Frame Slotted ALOHA, when a perfect channel is assumed. In more realistic channel environments, the use of SIC brings an important performance boost.
\end{abstract}

Keywords: Machine-to-machine (M2M) communications, Frame Slotted ALOHA, discrete-time Markov chain, performance evaluation

\section{Introduction}

A fundamental part of the Internet of Things (IoT) is the concept of machine-to-machine (M2M) communications, that allows the autonomous exchange of data between a large population of devices [1]. The rapid increase in the number of M2M devices deployed brings serious design challenges. One of these challenges is the efficiency of the Medium Access Control (MAC) protocol. The operation simplicity of random access protocols, such as ALOHA or Carrier Sense Multiple Access (CSMA), makes them a

\footnotetext{
This work has been supported by the Ministry of Economy and Competitiveness of Spain through projects TIN2013-47272-C2-1-R and TEC2015-71932-REDT. The authors would like to thank the support received from the Instituto ITACA (Instituto Universitario de Tecnologías de la Información y Comunicaciones) at the Universitat Politècnica de València, Spain. C. Portillo acknowledges the funding received from the European Union under the program Erasmus Mundus Partnerships, project EuroinkaNet, GRANT AGREEMENT NUMBER $2014-0870 / 001 / 001$, and the support received from SEP-SES (DSA/103.5/15/6629).

Email addresses: vcasares@upv.es (Vicente Casares-Giner ), jmartinez@upv.es (Jorge Martinez-Bauset), caporji@upv.es (Canek Portillo)
}

good choice for M2M communications. In addition, when the power consumption is of primary importance, a protocol of the ALOHA family may be a better choice than one of the CSMA family, as the sensing phase is missing [2].

Since the seminal work of the ALOHA protocol [3], and its slotted version S-ALOHA [4], many different extensions have been proposed. A variant named Frame SlottedALOHA (FSA), studied in detail in $[5,6,7]$, is worth mentioning. Due to its simplicity, FSA is implemented in different practical systems such as, Radio Frequency IDentification (RFID) [8]. Also, it has been proposed for low power wide area networks (LPWAN) [9] and M2M communications [10], to name just a few examples.

A common operation feature in many of these systems is that FSA is used to transmit only data packets (DAP). However, when a collision occurs the entire data slot is lost. A more efficient approach is to use short reservation packets (RVP) for contention, and allocate reserved data slots for those devices that succeeded with the reservation (their RVP did not collide). In this way, when RVP collide, only reservation mini-slots are lost, instead of the more valuable data slots. We call this protocol FSA with reservation and data packets (FSA-RDP). 
FSA-RDP is different to other S-ALOHA reservation protocols like R-ALOHA [11]. R-ALOHA was proposed for scenarios where devices have to transmit long messages, that are divided into shorter packets. The contention is solved using data (packet) slots. Once a device successfully sends the first DAP of the message, data slots are reserved in subsequent frames for the transmission of the rest of the DAP that compose the message. Then, FSA-RDP could also be used to improve the performance of R-ALOHA.

One of the main contributions of this paper is the analytical performance evaluation of the FSA-RDP protocol. For the M2M devices, we consider the FIFO - first in, first out - service discipline with two queue management schemes, tail drop (TD) and push-out (PO) [12]. When TD is employed, a new DAP is lost if it arrives to a M2M device with a full buffer. However, when PO is employed, the arrival of a new DAP pushes-out the DAP stored at the head of the full buffer. For M2M devices with a buffer size of one DAP we develop a discrete-time Markov chain (DTMC) to determine the DAP delay distribution and other performance parameters.

A preliminary analytical study of the FSA-RDP protocol was presented in [13]. In the study presented here we provide additional analytical details of the model, we describe its operation thoroughly, and provide results that validate its solution. Additional contributions of the new study are: i) we extend the previous study including buffer sizes larger than one; ii) we provide evidences of the interest of introducing an adaptive scheme that sets the permission probability and the number of reservation mini-slots per frame according to the offered load; iii) we analyze scenarios with imperfect channel, where the controller implements successive interference cancellation (SIC). We provide new results that compare the performance of systems that implement SIC to those that do not implement SIC. In addition, we evaluate the energy efficiency; iv) we study the impact of using Irregular Repetition Slotted ALOHA (IRSA) at the devices to send RVP when a perfect channel is assumed, and evaluate the energy efficiency.

The paper is organized as follows. After the introduction, Section 2 provides a comprehensive overview of past ALOHA contributions. We highlight the merits and shortcomings of previous proposals. A description of our contributions and the motivations of our work is presented in Section 3. Section 4 describes the basic operation of the system under study, and introduces the DTMC that defines the evolution of the number of active devices with time. In Section 5 the probability generating functions of the delay distribution for successfully transmitted DAP are derived, both for the TD and PO queue management schemes. In Section 6 the analytical model is validated by extensive simulations. Also, a study of the efficiency of the FSA-RDP protocol is discussed. Results for the channel utilization, cumulative distribution function of the data packet delay, average delay, 95-th percentile of the data packet delay are also obtained when devices have buffer sizes of one, five and ten DAP. Finally, Section 6 also presents performance results of scenarios where SIC and IRSA are implemented. The conclusions of the study are described in Section 7.

\section{Related Work}

Random Access Protocols (RAP) are commonly implemented in wired and wireless access networks. RAP are inherently unstable, so stabilization procedures must be seriously considered before being used in practical implementations. The stabilization procedure for RAP is implemented by combining two types of protocols or algorithms, channel access algorithms (CAA) and contention resolution algorithms (CRA) [14]. According to [15], they can also be characterized as global and local strategies, respectively. While CAA define rules by which new DAP access the channel, CRA indicate how collisions are resolved. Most of the known CAA and CRA appear in isolation, although sometimes they join forces with each other [16].

Three main CAA categories have been considered in the literature. One is the free channel access, where new DAP are immediately transmitted in the first slot following their arrival. They are sometimes referred to as immediate first transmission (IFT). In other words, new and backlogged DAP (those retrying) are treated identically [17].

A second category is the gated channel access, in which new arrivals delay their first transmission attempt until the contention resolution interval (CRI) during which they arrived has end. A pioneer example was proposed in [18].

A third category is the window channel access, in which time is divided into consecutive intervals of $\Delta_{i}$ seconds, $i=1,2, \ldots$ A DAP that arrives during $\Delta_{i}$ produce the $i-t h \mathrm{CRI}$, i.e., it accesses during that CRI. The $\Delta_{i}$ time intervals can be adapted to traffic conditions. A window with constant duration, $\Delta_{i}=\Delta \forall i$, has also been adopted [19, 20]. Both, gated channel access and window channel access are also collectively referred to as delayed first transmission (DFT) [17].

Two main CRA have been treated extensively in the literature. First, splitting algorithms are based on splitting a given conflict into smaller ones. Splitting algorithms offer different implementations, see [21].

Second, another approach is to treat all devices involved in a conflict identically by defining a common transmission or permission probability for both new and backlogged devices. Within this second subset of algorithms we can find the network centralized control by Bayesian broadcast probability proposed in [17].

The maximum stable throughput reachable by a RAP depends on the stabilization procedure used. The system throughput is defined as the average number of successful DAP transmitted per time-slot. To evaluate the throughput, as well as the packet delay, several aspects must be taken into account. For example, channel characterization, conflict feedback, full or limited feedback when sensing the channel, DAP arrival process and buffer size per device. 
Most of the proposed analytical models assume a perfect channel with no errors, negligible propagation delay, and no capture effect when a collision occurs. Also, an instantaneous ternary (E/S/C, E:empty, S:success, C:collision) or binary (C/No $\mathrm{C}, \mathrm{S} / \mathrm{No} \mathrm{S})$ feedback is quite often assumed [14]. With respect to the arrival process, for tractability, the standard approach is to assume a Poisson (Bernoulli) arrival process for infinite (finite) number of devices. However, some studies propose different arrival processes, such as D-BMAP [14].

In the following examples it is assumed that the channel capacity is unity, and DAP fit into a single slot. Then, the throughput expressed in DAP per time-slot and the channel utilization coincide. In [17], with E/S/C feedback and with the IFT policy, the stability of the RAP is guaranteed for $\lambda<1 / e \approx 0.3678$, when DAP arrivals follow a Poisson process with rate $\lambda \mathrm{DAP} /$ slot. The splitting algorithm proposed in [18] and [20] consider a window channel access (CAA with DFT) and it is stable for $\lambda<0.4295$.

\subsection{Frame Slotted ALOHA}

Frame Slotted ALOHA (FSA) is an important family of RAP [5]. FSA can be seen as a generalization of the standard S-ALOHA. In FSA a set of $F$ consecutive slots conforms a frame, $F>1$. At the beginning of a frame, each device that has a DAP to transmit selects one of the $F$ time-slots with equal probability. If the device does not receive an acknowledgment from the central controller, it will try again in the next frame.

FSA and its variants have been extensively evaluated in the literature. One example is the study in [7], where an exact performance evaluation analysis of FSA with capture is presented. The study assumes a finite number of unbuffered devices (i.e., can hold at most one DAP at a time), and a Bernoulli traffic model. Another example is the work in [22], where a dynamic FSA (DFSA) is proposed. Here, the frame length $F$ is estimated according to the expected value of the number of backlogged DAP at the beginning of a frame. Under the assumptions of a Poisson arrival process, single DAP per message, perfect channel and no capture effect, the proposed DFSA achieves a maximum throughput of 0.426 . Clearly, it is slightly higher than the one achieved by an stabilized S$\operatorname{ALOHA}(1 / e \approx 0.3678)$.

We highlight the fact that the maximum achievable throughput in FSA with infinite number of sources, Poisson arrival process, and without dynamic frame length adaptation, equals the one achieved by the ordinary SALOHA [5].

\subsection{FSA with Multiple Packet Replicas and Interference Cancellation}

The throughput of the FSA protocol can be enhanced if multiple copies (replicas) of the same data packet are transmitted in the same frame. In [23], the authors present a pioneering scheme called DSA (Diversity Slotted ALOHA) for satellite communications. The idea is to transmit several copies of the same packet at different time-slots. They found that under light traffic, multiple transmission gives better delay performance. Also, mainly for satellite communications, the CRDSA method is proposed in [24]. There, two replicas of the same DAP are sent in the same frame, but in different time-slots. A correct reception of a DAP (without collision) in a given time-slot can be exploited when a interference cancellation (IC) technique is implemented, enabling the recovery of other DAP that initially could not be decoded. The implementation of the CRA in CRDSA is based on IC, a technique that requires a good estimation of the channel for the elimination of collision bursts. With CRDSA the achieving maximum throughput is 0.55 , approximately. In [25], the number of DAP replicas is no longer limited to two. Its number is chosen randomly by the device at each frame. The scheme, referred to Irregular Repetition Slotted ALOHA (IRSA) achieves a throughput around 0.97 for large frames, $F>>1$, and around 0.8 in practical implementations.

The SICTA algorithm is presented in [26], where a cross layer design approach is proposed assuming a unit frame length $(F=1)$. To resolve collisions, SICTA uses successive IC (SIC) and is combined with a tree algorithm as a CRA algorithm. SICTA achieves a maximum throughput of 0.6931 , when a binary tree is implemented.

In [27] the coded-S-ALOHA (CSA), an extension of the IRSA access strategy is proposed. In addition to the use of DAP replicas, DAP are encoded prior to transmission, for example using a linear block code which, in general, is different among users. Then, IRSA can be seen as a particular case of CSA, where all replicas can be interpreted as repetition codes. Assuming a perfect channel, the authors of [28] show the benefit of using SIC with FSA when a random number of replicas is implemented, and both intra-frame and inter-frame relationships are considered.

The physical layer corresponding to the previous access techniques is based on the temporal or frequency orthogonality, i.e., TDMA or CDMA, respectively. They are recognized as orthogonal multiple access (OMA) schemes. Non-orthogonal multiple access (NOMA) schemes such as CDMA, turn out to be less restrictive than OMA schemes to approximate Shannon limits on imperfect communication channels. Interleaved Division Multiple Access (IDMA) is a solution for RA within the family of OMA schemes $[29,30]$. In IDMA different terminals share the same bandwidth and time-slot, and their transmissions can be separated and decoded correctly in the receiver when implementing an iterative interference cancellation (IIC) algorithm.

In [31] the authors propose the Scrambled Coded Multiple Access (SCMA) method, where scrambling sequences replace the long interleaved tables used in IDMA, and bring some additional advantages. The Asynchronous SCMA (A-SCMA) introduced in [32] assumes that the transmission from different terminals are not synchronized. In this case, the multi-user cancellation algorithm is im- 
plemented to recover the information that appears partially overlapped. In [33] the authors propose an extension of their own work [32], by adding spreading to A-SCMA (SA-SCMA). They show that even using modest spreading values, significant gains in spectral efficiency are achieved.

In [34] a new random access scheme is proposed named Segmented FSA (SFSA). In SFSA, each packet is encoded and subdivided into segments before the transmission. To improve the success probability IC is adopted when segment collisions occur. In [35] an extensive and comprehensive survey of RA techniques is provided, mainly for satellite applications.

\subsection{Reservation Schemes}

S-ALOHA and FSA protocols can be used to transfer messages composed by multiple packets. One pioneering example is R-ALOHA, proposed to improve the throughput of satellite communications [11]. Here, the contention is solved using data (packet) slots. Once a device successfully sends the first packet of the message, data slots are reserved in subsequent frames for the transmission of the rest of the packets that compose the message.

An improved protocol was proposed in [36], where frames are divided into contention mini-slots and data time-slots. Mini-slots are used for the out-of-slot signaling protocol (reservation), in opposition to in-slot signaling protocols where contention occurs in data time-slots [37]. Once a device succeeds with a reservation, it can transfer a packet in a reserved data slot, and the rest of the message in reserved data slots in subsequent frames.

Since then, extensive studies of reservation FSA (RFSA) have been conducted. Some representative examples are $[6,38,39]$. In [6], it is assumed that devices have buffers that hold a single packet, packets arrive according to a Bernoulli model, and out-of-slot signaling is proposed. Successful request are placed in a common FIFO queue of finite capacity. The exact analytical model provides computable formulas for delay and throughput.

In contrast, in [38] and [39] it is assumed that devices are equipped with buffers that hold a single multi-packet message, messages arrive according to a Bernoulli model, and in-slot signaling is proposed. At frame initiation, devices with a message to transmit randomly choose one of the non-reserved data slots. If the device succeeds with the transmission of the first packet, slots will be reserved to transmit the rest of the packets of the message in the following frames. Otherwise, it contends in the next frame. Clearly, these protocols operate with the IFT principle.

RFSA has been proposed and studied in [40, 10] for M2M applications. In [40] a finite number of devices each one with a multi-packet message ready to transmit try to gain access by means of the first data packet and according to the FSA protocol. Devices that successfully access, get a reserved slot in order to transmit the rest of the message in subsequent frames. RFSA has also been proposed and implemented in many other practical applications. For example, in unmanned aerial vehicles (UAVs) communications [41] and in vehicle communication with road side units [42].

\section{Motivation and Contributions of the Study}

We propose an analytical model to evaluate the performance of a FSA scheme in which mini-slots are used for reservation and data slots to transmit DAP (out-ofslot signaling). Frames are composed of a reservation subframe (RSF) and a data subframe (DSF). Typically, the RSF is divided into multiple mini-slots, that devices use for contention access following the FSA scheme. The DSF is composed of a variable number of data slots, the duration of each of them being multiple mini-slots. Once a device succeeds with a reservation, it transmits a single DAP in one data slot of the same frame. Then, the maximum number of data slots in a frame equals the number of reservation mini-slots. Note that occasionally it may happen that the DSF has zero data slots. Clearly, devices operate following the IFT principle.

Data packets arrive to devices according to any renewal arrival process, as the model characterizes the number of DAP that arrive to a device per mini-slot by independent and identically distributed random variables. Then, at each mini-slot, DAP arrive following a general distribution irrespective of where the DAP were originated, i.e., internally (as a consequence of its own sensing activity) or externally (arriving from another device). For simplicity, we assume that DAP arrive according to a Poisson process with a constant rate. Therefore, the number of DAP arrivals per mini-slot follows a discrete Poisson distribution.

As in $[6,7]$, for model tractability, we assume that devices are equipped with buffers that hold a single DAP. However, we analyze systems with buffer sizes larger than one by simulation. Also for tractability of the analytical model, we consider a perfect channel, with no capture effects, and instantaneous ternary feedback (E/S/C). Nonetheless, we analyze systems with imperfect channel and SIC by simulation.

To the best of authors knowledge, the proposed model is the first analytical model proposed in the literature to evaluate the performance of the Frame Slotted ALOHA with Reservation and Data Packets (FSA-RDP) at the minislot granularity. One of the main differences between the model proposed in [6] an the one proposed in our study is that the duration of the frame is variable, instead of being of fixed duration as in [6]. This is an important difference as it leads to a higher channel utilization. In addition, we consider two queue management schemes, tail drop (TD) and push-out (PO). We believe that PO schemes have an important role in scenarios where sensors report measurements of the environment. Here, the current measurement might be of higher value than past ones.

Finally, our analytical approach is completely different to the one in [6]. We exploit the more structured matrix geometric analysis, while the model in [6] is based 


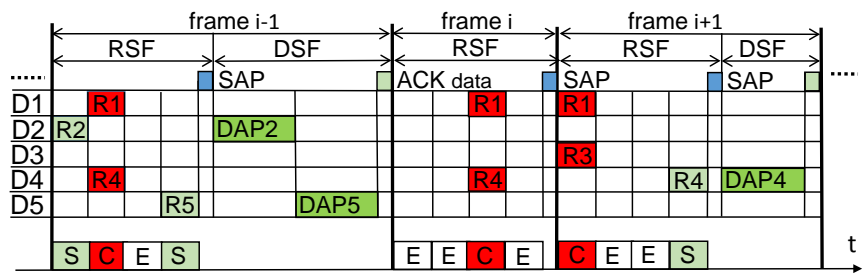

Figure 1: Example of the operation of the FSA-RDP protocol. S: success, C: collision, E: empty.

on conventional probabilistic arguments. In addition, the proposed model allows to determine the data packet delay distribution at the granularity of the mini-slot, while the model in [6] allows only to determine average delays at the granularity of the frame duration.

An ALOHA protocol that implements reservation minislots and data slots has been studied in [43] by simulation. However, FSA-RDP is different to the protocol studied there, where no DAP are allowed to enter the system until the controller fully resolves the collisions that occurred during the last access, using for this a distributed queue algorithm. That is, a DFT approach, in contrast to the IFT deployed in our work.

\section{System model}

\subsection{Protocol Operation}

We consider a wireless network composed by a controller (gateway) and a finite number of devices forming a star topology. Devices operate independently and transmits DAP of constant size as a consequence of their activity. In the RSF, active devices (with DAP in their queues) contend for channel access by transmitting RVP. At the end of the RSF, the controller broadcasts a slot allocation packet (SAP) that contains the data slots allocated to those devices that succeeded with the reservation. Following the SAP, successful devices initiate the transmission of one DAP per reserved data slot, i.e., in a contention free manner. Finally, the controller broadcast an control packet to acknowledge the successful reception of the DAP.

Figure 1 describes an example of the operation of FSARDP, where 5 devices transmit DAP to the controller. Observe that we denote by: i) $\mathrm{D} i$ the devices; ii) $\mathrm{R} i$ the RVP transmitted by D $i$; iii) DAP $i$ the DAP transmitted by D $i$; iv) $E$ empty reservation mini-slot; v) $S$ successful RVP; vi) $C$ collided RVP. At frame $i-1$ four devices contend for access sending their respective RVP during the RSF. $\mathrm{R} 2$ and R5 succeed, while R1 and R4 collide. At the end of the RSF, the controller broadcasts the SAP to allocate data slots to the successful devices. Then, D2 and D5 send DAP2 and DAP5 during the DSF. At frame $i, \mathrm{R} 1$ and R4 collide again. Then, the controller broadcasts the SAP informing that the DSF is omitted in this frame. At frame $i+1$, R1 and R3 collide, while R4 succeeds. Then, the DSF ends after DAP4 is transmitted. Observe that D3 was inactive at the beginning of frame $i-1$ and frame $i$, and received a DAP during frame $i$.

\subsection{Network Model}

Let $M$ be the number of devices that compose the wireless sensor network. DAP arrive to a device according to a Poisson process with rate $\lambda \mathrm{DAP} /$ mini-slot, and devices are equipped with a buffer that can hold a single DAP. Let $Q$ denote the buffer size in DAP, then $Q=1$.

Let $W$ be the duration of a data slot in mini-slots. Also, let $V$ be the number of reservation mini-slots in the RSF. It can be shown that the reservation success probability is maximized when the number of contenders in each RSF is $n_{\text {opt }}=(\ln (V /(V-1)))^{-1}[44]$. From the basic inequalities, $1-1 / x<\ln (x)<x-1$, for $x>0$, it follows immediately that $V-1<n_{\text {opt }}<V$. This suggest than the number of reservations mini-slots $V$ per frame should be adapted to the number of estimated contenders. However, for model tractability, we consider a fixed value for $V$.

We introduce an access permission probability $r$ [17]. Then, at each RSF, active devices send a RVP with probability $r$. Note that $r$ can be adjusted by the controller according to the observed outcomes of the previous RSF, and the estimated number of DAP arrivals during the whole frame. The adaptive algorithm is left for further study. Then, we also consider a fixed value for $r$.

Let $i$ be the number of active devices at the beginning of a RSF. The probability that $j$ of them, $j \leq i \leq M$, transmit a RVP follows a binomial distribution, and denote it as $B_{j}^{i}(r)$. A device selects any of the $V$ mini-slots to transmit the RVP with equal probability $(1 / V)$.

Let $S_{k}^{j}(V)$ denote the probability that $k$ among $j$ contending devices succeed with the reservation. It can be obtained recursively as,

$$
\begin{aligned}
S_{k}^{j}(V) & =\sum_{n=0, \neq 1}^{j} B_{n}^{j}(1 / V) S_{k}^{j-n}(V-1), \quad k=0 \\
S_{k}^{j}(V) & =B_{1}^{j}(1 / V) S_{k-1}^{j-1}(V-1) \\
& +\sum_{n=0, \neq 1}^{j-k} B_{n}^{j}(1 / V) S_{k}^{j-n}(V-1), \quad k=1, \ldots, V
\end{aligned}
$$

Recurrence (1) is based on the following sequential reasoning. First, we observe the contention outcome in the first mini-slot of the RSF: empty (no RVP), success (a single RVP), or collision (multiple RVP). Based on this outcome, we formulate the contention outcome of the remaining mini-slots of the RSF.

Similar recursive algorithms have been proposed in the literature. See for instance equation (12) in [22]. Also, in [45] a new recursive scheme is proposed to evaluate the joint distribution of the number of successful and collided packets. The authors study the computational complexity of different algorithms, and show that the one they propose is quite efficient, even for large number of devices 
and reservation mini-slots $(V)$. From the joint distribution, marginal distributions can be readily determined.

Let $D_{k}^{i}(r, V)$ denote the probability that $k$ among $i$ active devices succeed in a frame, i.e., their RVP do not collide. It is given by,

$$
D_{k}^{i}(r, V)=\sum_{j=k}^{i} B_{j}^{i}(r) S_{k}^{j}(V), \quad 0 \leq k \leq j \leq i .
$$

The evolution of the number of active devices observed at the beginning of each RSF can be modeled as a DTMC. Let $\left\{P_{i j}\right\}$ denote the transition probabilities of the DTMC, i.e., the probability of $j$ active devices in the next frame $n+1$, conditioned on $i$ active ones in the current frame $n$. They are given by,

$$
P_{i j}=\sum_{k=\max (0, i-j)}^{\min (i, V)} D_{k}^{i}(r, V) A_{j-i+k}^{M-i+k}\left(a_{k}\right),
$$

where $A_{u}^{s}\left(a_{k}\right)$ is the probability that $u$ devices become active among $s$ inactive in a frame with $k$ successful reservations, $a_{k}$ is the probability that at least one DAP arrives to a device during a frame of duration $t_{k}$ mini-slots, $t_{k}=V+k W, 0 \leq k \leq V$. That is, $a_{k}$ reflects the individual activity of each devices. For Poisson arrivals, $a_{k}=1-e^{-\lambda t_{k}}=1-\delta^{t_{k}}$ (for convenience, we define $\left.\delta=e^{-\lambda}\right)$. Clearly, $A_{u}^{s}\left(a_{k}\right)$ follows a binomial distribution.

The definition of $t_{k}$ allows the model to determine the delay of DAP at the granularity of a mini-slot. Also, notice that since we assume $Q=1$, no more than one DAP per frame and per device can be admitted. Finally, we point out that both, the transmission of a SAP at the end of a $\mathrm{RSF}$, and the transmission of an ACK at the end of a DSF by the controller, require a single mini-slot. For simplicity, this constant delay term has been omitted in the analysis.

Then, with TD scheme, the first DAP that arrives along frame $n$ (the new DAP) is transferred to the buffer for transmission if it is found empty. If the buffer is occupied with a previous DAP that succeed in the $\operatorname{RSF} n$, the new DAP also joins the buffer. Otherwise, the new DAP is dropped. However, with PO, the last DAP that arrives along a frame $n$ (the new DAP), is transferred to the buffer for transmission regardless of the state of the buffer. In particular, if the buffer is found occupied with a previous DAP that did not succeed in the $\operatorname{RSF} n$, this DAP is pushed-out by the new one.

Let $\pi_{i}$ denote the stationary probability of finding $i$ active devices at the beginning of an arbitrary RSF. The stationary probabilities $\boldsymbol{\pi}=\left[\pi_{0}, \pi_{0}, \ldots, \pi_{M}\right]$ are obtained by solving the system of linear equations $\boldsymbol{\pi}=\boldsymbol{\pi} \mathbf{P}$ with the normalization condition of $\boldsymbol{\pi} \mathbf{e}=1$, where $\mathbf{P}=\left[P_{i j}\right]$, and $\mathbf{e}$ is a column vector of $1 \mathrm{~s}$. Then, the throughput $\gamma$ (carried DAP rate), in DAP per mini-slot, can be determined by,

$$
\gamma=\sum_{k=0}^{V} k f_{k} / \sum_{k=0}^{V} t_{k} f_{k}, \quad f_{k}=\sum_{n=k}^{M} \pi_{n} D_{k}^{n}(r, V)
$$

where $f_{k}$ is the fraction of frames which duration is equal to $t_{k}=V+k W$.

\section{Data Packet Delay Distribution}

Let us consider a frame randomly chosen, frame $n$, and suppose that its duration is $t_{m}=V+m W$. We assume that our tagged device receives at least one DAP during that frame; this occurs with probability $a_{m}$. Note that only one DAP can be hold at the device buffer $(Q=1)$. We observe the number of active devices at the beginning of that frame. A device is active when has a DAP in its buffer. Clearly, since the frame has a duration $t_{m}$, at least $k$ devices, $m \leq k \leq M$, were active at the beginning of that frame $(M-k$ were inactive). Our tagged device could be one of the active devices or not. In the following Subsections we distinguish three different cases.

\subsection{Case $A$}

At the beginning of frame $n$, the tagged device is not active, i.e., it is not among the $k$ active ones. This occurs with probability $(M-k) / M$. Assuming that $m$ out of $k$ terminals gain access in the $\operatorname{RSF} n$, the probability that our tagged terminal will compete in the next frame $n+1$ with other $j$ terminals, $0 \leq j \leq M^{\prime}=M-1$, is given by,

$$
P_{A}(M, m, j, k)=\frac{M-k}{M} D_{m}^{k}(r, V) A_{j-k+m}^{M^{\prime}-k+m}\left(a_{m}\right) .
$$

Weighting the above expression by the steady state probabilities $\pi_{k}, m \leq k \leq M$, we have,

$$
P_{A}(M, m, j)=\sum_{k=m}^{M} \pi_{k} P_{A}(M, m, j, k) .
$$

Since the probability that our tagged terminal receives at least one DAP during the frame $n\left(a_{m}\right)$ depends on the frame duration $t_{m}$, (6) must be weighted by $a_{m}$. Furthermore, in order to include the arrival instant of the DAP within frame $n$ we have to distinguish between the TD and the PO schemes. In particular, respectively we have,

$$
\begin{gathered}
F_{m}(z)=\sum_{l=0}^{t_{m}-1} \delta^{t_{m}-1-l}(1-\delta) z^{l}, \\
L_{m}(z)=\sum_{l=0}^{t_{m}-1}(1-\delta) \delta^{l} z^{l} .
\end{gathered}
$$

Both expressions (7) and (8) are generating functions that account for the admitted DAP arriving at any minislot of the frame $n$. Obviously, $a_{m}=F_{m}(z=1)=L_{m}(z=$ $1)$. They are explained as follows. In (7), we keep in mind that the duration of frame $n$ is $t_{m}$ mini-slots. Then, for $l=0$, a DAP arrives to the tagged device during the last mini-slot of frame $n$, and no DAP arrive during the previous mini-slots. This occurs with probability $\delta^{t_{m}-1}(1-\delta)$. 
For $l=1$, a DAP arrives to the tagged device in the penultimate mini-slot of frame $n$, and no DAP arrive during the previous mini-slots. This occurs with probability $\delta^{t_{m}-2}(1-\delta)$. Note that the potential arrival of one DAP during the last mini-slot has no effect, as this DAP is rejected because the TD scheme is being considered. For $l=2,3, \ldots, t_{m}-1$ the same reasoning applies.

For expression (8) we argue as follows. For $l=0$, with probability $(1-\delta)$ a DAP arrives to the tagged device during the last mini-slot, and pushes-out previous arrivals, if any. For $l=1$, with probability $(1-\delta)$ a DAP arrives to the tagged device during the penultimate mini-slot and pushes-out previous arrivals, if any. It will contend in the RSF $n+1$ if no arrivals occur during the last mini-slot. This occurs with probability $(1-\delta) \delta$. This line of reasoning can be extended to the rest of mini-slots.

Also, the normalization with respect to the frequency of occurrences of intervals of duration $t_{m}$ weighted by $a_{m}$ is required. Then, we can write,

$$
P_{A, X}(M, j, z)=\left(1 / G_{a}\right) \sum_{m=0}^{V} X_{m}(z) P_{A}(M, m, j),
$$

where $G_{a}=\sum_{i=0}^{V} a_{i} f_{i}$ and $X=F, L$; i.e. $X_{m}(z)=$ $F_{m}(z), L_{m}(z)$, for TD or PO schemes, respectively.

\subsection{Case $B$}

At the beginning of the frame $n$, the tagged device is active, i.e., it is one of the $k$ active devices. This situation occurs with probability $k / M$. Assuming that $m$ out of $k$ devices succeed with the reservation, the tagged device succeeds with probability $m / k$. Then, the DAP preceding our tagged DAP is successfully transmitted in the actual frame $n$. The tagged DAP is copied to the buffer as soon as the previous DAP in the buffer is transmitted. In that case, the probability that our tagged device will compete in the next frame $n+1$ with other $j$ active devices, $0 \leq$ $j \leq M^{\prime}=M-1$, is given by,

$$
P_{B}(M, m, j, k)=\frac{k}{M} \frac{m}{k} D_{m}^{k}(r, V) A_{j-k+m}^{M^{\prime}-k+m}\left(a_{m}\right)
$$

As in (6), weighting the above expression by the steady state probabilities $\pi_{k}, m \leq k \leq M$ we have

$$
P_{B}(M, m, j)=\sum_{k=m}^{M} \pi_{k} P_{B}(M, m, j, k) .
$$

Finally, in the same way as in (9), weighting the expression (11) we get,

$$
P_{B, X}(M, j, z)=\left(1 / G_{a}\right) \sum_{m=0}^{V} X_{m}(z) P_{B}(M, m, j) .
$$

\subsection{Case $C$}

This case complements Case $B$, where we assumed that the tagged device was active. In addition, we assume here that the tagged device does not succeed with the reservation in frame $n$. This occurs with probability $(k-m) / k$. Then the tagged DAP that arrives along frame $n$ will be lost with the TD scheme $(Q=1)$, but admitted with the PO scheme (as it pushes-out the DAP in the buffer). In this later case, the probability that the tagged DAP contends with other $j$ active devices in frame $n+1$, $0 \leq j \leq M^{\prime}=M-1$, is given by,

$$
P_{C}(M, m, j, k)=\frac{k}{M} \frac{k-m}{k} D_{m}^{k}(r, V) A_{j-k+m+1}^{M-k+m}\left(a_{m}\right) .
$$

Then, as in (6) and in (11), weighting the above expression by the steady state probabilities $\pi_{k}, m \leq k \leq M$, we have,

$$
P_{C}(M, m, j)=\sum_{k=m}^{M} \pi_{k} P_{C}(M, m, j, k)
$$

In the same way as in (9) and in (12), weighting the expression (14) we get,

$$
\left.P_{C, P O}(M, j, z)\right)=\left(1 / G_{a}\right) \sum_{m=0}^{V} L_{m}(z) P_{C}(M, m, j)
$$

\subsection{Phase-Type Distributions}

The delay distribution of successfully transmitted DAP under the TD and PO buffer management scheme follow Phase-type $(\mathrm{PH})$ distributions. They are represented as $\left(\boldsymbol{\alpha}, \mathbf{T}, \mathbf{T}^{0}\right)$, where $\boldsymbol{\alpha}$ defines the probabilities that the absorbing process is initiated at each of its transient states, $\mathbf{T}$ defines the transition probabilities between transient states, and $\mathbf{T}^{0}=\mathbf{e}-\mathbf{T e}$ is a column vector that defines the transition probabilities from transient states to the absorbing state [46, 47].

For the TD and PO schemes we denote them as $\left(\boldsymbol{\alpha}_{T D}, \mathbf{T}_{T D}, \mathbf{T}_{T D}^{0}\right)$, and $\left(\boldsymbol{\alpha}_{P O}, \mathbf{T}_{P O}, \mathbf{T}_{P O}^{0}\right)$, respectively.

The initial probability vectors for the $\mathrm{PH}$ distributions follow from (9), (12) and (15). For TD we have, $\boldsymbol{\alpha}_{T D}(z)=$ $\left[\alpha_{T D, 0}(z), \ldots, \alpha_{T D, M^{\prime}}(z)\right]$, and for PO scheme, $\boldsymbol{\alpha}_{P O}(z)=$ $\left[\alpha_{P O, 0}(z), \ldots, \alpha_{P O, M^{\prime}}(z)\right]$, where

$$
\begin{aligned}
\alpha_{T D, j}(z) & =P_{A, T D}(M, j, z)+P_{B, T D}(M, j, z) \\
\alpha_{P O, j}(z) & =P_{A, P O}(M, j, z)+P_{B, P O}(M, j, z) \\
& +P_{C, P O}(M, j, z)
\end{aligned}
$$

where $\boldsymbol{\alpha}_{T D}(1) \mathbf{e}<1$, i.e., $\boldsymbol{\alpha}_{T D}(1)$ is a substochastic vector, and $\boldsymbol{\alpha}_{P O}(1)$ is a stochastic vector, $\boldsymbol{\alpha}_{P O}(1) \mathbf{e}=1$. 


\subsection{Matrix $\mathbf{T}_{T D} \mid \mathbf{T}_{T D}^{0}$ for TD scheme}

Let $T_{T D, k ; i, j}$ be the probability that the tagged device contends with other $i$ active devices in a frame, $k$ devices succeed with the reservation, but the tagged device does not succeed, and contends in the next frame with $j$ other active devices. Then, for $0 \leq i, j \leq M^{\prime}=M-1$ and $0 \leq k \leq i+1$

$$
\begin{aligned}
& T_{T D, k ; i, j}=\left(1-\frac{C_{k-1}^{i}}{C_{k}^{i+1}}\right) D_{k}^{i+1}(r, V) A_{k-i+j}^{M-i-1+k}\left(a_{k}\right) \\
& \quad=\left(1-\frac{k}{i+1}\right) D_{k}^{i+1}(r, V) A_{k-i+j}^{M-i-1+k}\left(a_{k}\right)
\end{aligned}
$$

where $C_{j}^{i}=\left(\begin{array}{l}i \\ j\end{array}\right)$. In matrix notation and in terms of the probability generating function (pgf) we have,

$$
\mathbf{T}_{T D}(z)=\sum_{k=0}^{V} \mathbf{T}_{T D, k} z^{(V+k W)} .
$$

To get a better understanding of (17) we remember that, when $k$ devices succeed with the reservation, the RSF is followed by a DSF of duration $k W$ mini-slots.

Let $T_{T D, k ; i}^{0}$ be the probability that the tagged device contends with other $i$ active devices in a frame, $k$ devices succeed with the reservation, and the tagged device is one of them. It is given by,

$$
T_{T D, k ; i}^{0}=\frac{C_{k-1}^{i}}{C_{k}^{i+1}} D_{k}^{i+1}(r, V)=\frac{k}{i+1} D_{k}^{i+1}(r, V) .
$$

Let $\mathbf{T}_{T D, k}^{0}$ denote the column vector with elements given by (18). Again, in matrix notation and in terms of the generating functions, we have the following column vectors,

$$
\mathbf{T}_{T D}^{0}(z)=\sum_{k=0}^{V} \mathbf{T}_{T D, k}^{0} z^{(V+k W)} .
$$

In the above expression, it is assumed that the controller will send the ACK at the end of the DSF to acknowledge the successful reception of DAP to the $k$ corresponding devices. Note that once the tagged DAP is successfully transmitted in a given frame, the arrival of new DAP has no impact on the tagged DAP. That is, the term $A_{u}^{s}\left(a_{k}\right)$ is no longer needed in (18), but was needed in (16).

\subsection{Matrix $\mathbf{T}_{P O} \mid \mathbf{T}_{P O}^{0}$ for PO scheme}

Let $T_{P O, k ; i, j}$ be defined as (16) but with the additional condition that no DAP will arrive to the tagged device during a frame of duration $t_{k}$. Then,

$$
T_{P O, k ; i, j}=\left(1-a_{k}\right) T_{T D, k ; i, j} .
$$

Let $\mathbf{T}_{P O, k}$ denote a matrix with elements given by (20). In matrix notation and in terms of the pgf we have,

$$
\begin{aligned}
& \mathbf{T}_{P O}(z)= \\
& \sum_{k=0}^{V} \mathbf{T}_{P O, k} z^{(V+k W)}=\sum_{k=0}^{V}\left(1-a_{k}\right) \mathbf{T}_{T D, k} z^{(V+k W)} .
\end{aligned}
$$

With regard to $T_{P O, k ; i}^{0}$, the PO counterpart of (18), we have two contributions. The first one contains the probability that the tagged DAP is pushed-out in a frame of duration $t_{k}$, before it succeeds with a reservation. This happens with probability $a_{k} \sum_{j=0}^{M^{\prime}} T_{T D, k ; i, j}=a_{k} T_{T D, k ; i}$. The second contribution is coincident with the TD scheme, so with $T_{T D, k ; i}^{0}$ given in (18), and reflects the probability that the RVP of the tagged device does not collide, and the corresponding DAP is successfully transmitted in a frame of duration $t_{k}$. Then,

$$
T_{P O, k ; i}^{0}=T_{T D, k ; i}^{0}+a_{k} T_{T D, k ; i},
$$

and the parallel expression to (19) is

$$
\mathbf{T}_{P O}^{0}(z)=\mathbf{T}_{T D}^{0}(z)+\sum_{k=0}^{V} a_{k} \mathbf{T}_{T D, k} z^{(V+k W)} .
$$

\subsection{Data packet delay distributions}

Based on previous section, here we derive the pgf of the delay distribution of transmitted DAP for TD and PO schemes. Using the results given in (17) and (19), (21) and (23), then generating function of the sojourn time for TD is given by,

$$
\begin{aligned}
& R_{T D}(z)=\alpha_{T D, M} \\
& +\boldsymbol{\alpha}_{T D}(z)\left[\mathbf{I}+\mathbf{T}_{T D}(z)+\mathbf{T}_{T D}^{2}(z)+\ldots\right] \mathbf{T}_{T D}^{0}(z) \\
& =\alpha_{T D, M}+\boldsymbol{\alpha}_{T D}(z)\left[\mathbf{I}-\mathbf{T}_{T D}(z)\right]^{-1} \mathbf{T}_{T D}^{0}(z) \\
& =R_{T D, a}(z)+R_{T D, r}(z) .
\end{aligned}
$$

The identifications in $(24)$ are, $R_{T D, r}(z)=\alpha_{T D, M}>0$ as the probability that a DAP is rejected by the tagged device, and $R_{T D, a}(z)$ takes into account the delay distribution of the DAP accepted by the tagged device. Obviously $R_{T D}(1)=1$.

For $\mathrm{PO}$ we have, with $\alpha_{P O, M}=0$,

$$
\begin{aligned}
R_{P O}(z)= & \boldsymbol{\alpha}_{P O}(z)\left[\mathbf{I}+\mathbf{T}_{P O}(z)+\mathbf{T}_{P O}^{2}(z)+\ldots\right] \mathbf{T}_{P O}^{0}(z) \\
= & \boldsymbol{\alpha}_{P O}(z)\left[\mathbf{I}-\mathbf{T}_{P O}(z)\right]^{-1} \mathbf{T}_{P O}^{0}(z) \\
= & \boldsymbol{\alpha}_{P O}(z)\left[\mathbf{I}-\mathbf{T}_{P O}(z)\right]^{-1} \\
& {\left[\mathbf{T}_{T D}^{0}(z)+\sum_{k=0}^{V} a_{k} \mathbf{T}_{T D, k} z^{(V+k W)}\right] } \\
= & R_{P O, a}(z)+R_{P O, r}(z) .
\end{aligned}
$$

where $R_{P O, a}(z)$ and $R_{P O, r}(z)$ can be identified from (23). $R_{P O, a}(z)=\boldsymbol{\alpha}_{P O}(z)\left[\mathbf{I}-\mathbf{T}_{P O}(z)\right]^{-1} \mathbf{T}_{T D}^{0}(z)$ takes into account the delay distribution of the DAP that are transmitted before being pushed-out. $R_{P O, r}(z)$ takes into account the waiting time of those DAP that are pushed-out.

We are only interested in finding the delay for DAP that are finally transmitted. That is,

$$
R_{T D, a}(z)=\frac{\boldsymbol{\alpha}_{T D}(z)\left[\mathbf{I}-\mathbf{T}_{T D}(z)\right]^{-1} \mathbf{T}_{T D}^{0}(z)}{R_{T D, a}(1)},
$$


Table 1: Model Symbols and Parameters

\begin{tabular}{lcc}
\hline Input Parameters & Symbol & Value \\
\hline Number of devices & $M$ & 8 \\
Numb. reserv. mini-slots & $V$ & $1,2, \ldots$ \\
Permission probability & $r$ & {$[0,1]$} \\
Device buffer size & $Q$ & $\{1,5,10\}$ \\
Data packet duration & $W$ & mini-slots \\
Frame duration (mini-slots) & $t_{k}$ & $V+k W$ \\
Packet arrival rate & $\lambda$ & packet $/$ mslot \\
Device $i$ load & $\rho_{i}$ & $\lambda W$ \\
System load & $\rho_{T}$ & $M \rho_{i}$ \\
\hline Performance parameters & Symbol & Value \\
\hline Station.prob. $k$ active devices & $\pi_{k}$ & {$[0,1]$} \\
Fraction frames of duration $t_{k}$ & $f_{k}$ & {$[0,1]$} \\
System throughput & $\gamma$ & packet/mslot \\
Channel utilization & $S$ & {$[0,1]$} \\
Packet loss probability & $P_{L}$ & {$[0,1]$} \\
Packet transmission delay & $D$ & mini-slots \\
95-th percentile of $D$ & $D_{95}$ & mini-slots \\
Frac. frames a device inactive & $P_{I}$ & {$[0,1]$} \\
\hline
\end{tabular}

and

$$
R_{P O, a}(z)=\frac{\boldsymbol{\alpha}_{P O}(z)\left[\mathbf{I}-\mathbf{T}_{P O}(z)\right]^{-1} \mathbf{T}_{T D}^{0}(z)}{R_{P O, a}(1)}
$$

with $R_{T D, a}(1)=R_{P O, a}(1)=1-\alpha_{T D, M}$.

\section{Numerical results}

Different performance parameters are studied, such as the protocol efficiency measured in terms of the data packet loss probability. Also, we determine the channel utilization, the data packet throughput, the cumulative distribution function (CDF) of the data packet delay for packets that are successfully transmitted, and from it the 95-th percentile of the data packet delay.

We first validate the analytical model for $Q=1$ by comparing its performance results with those obtained by simulation. The simulation results are obtained by implementing the contention schemes, scheduling schemes, queue management schemes, and data transmission procedure in a custom-built $\mathrm{C}$ based discrete-event simulation program. The developed simulation model mimics the physical behavior of the MAC protocol considered (FSA$\mathrm{RDP})$. That is, in each frame a device receives DAP according to a given discrete distribution, contends for channel access with other devices using RVP when it is active (has DAP in the buffer), and, if it succeeds, then transmits a DAP in the data slot allocated by the controller. The simulation model also supports imperfect channels and SIC, when required.

The simulation results are completely independent of those obtained by the analytical model. That is, the computation of performance metrics by the simulation model is not dependent on the derived mathematical expressions at all, nor are the state transition probabilities used in these computations. The performance results reported are the

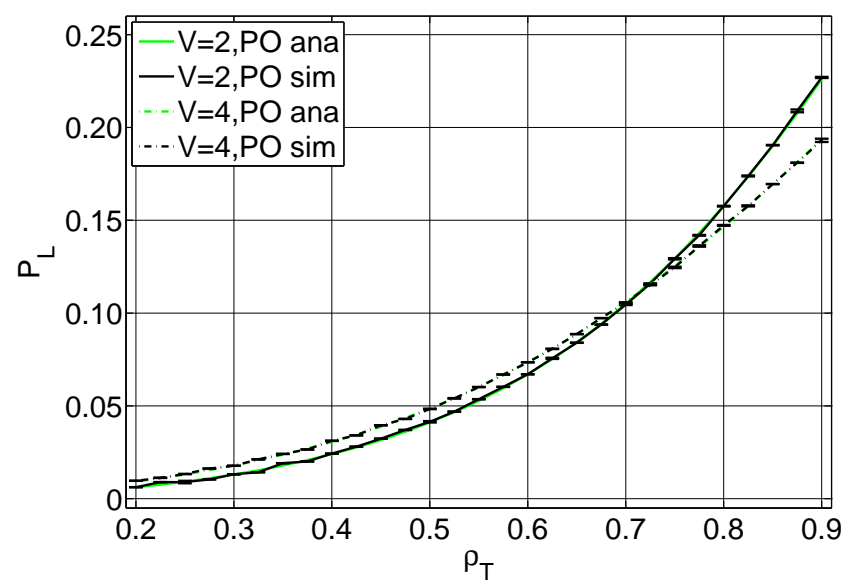

Figure 2: Analytical and simulation data packet loss probability with the offered load $\rho_{T}$.

average values of measurements made over $20 \cdot 10^{6}$ DAP arrivals. In addition, $95 \%$ confidence intervals have been obtained. However, as they are very small, and therefore not significant, they are only shown in Fig. 2 and 3 for illustration purposes, and omitted in the rest of figures.

We define a reference scenario where $M=8, W=10$, $Q=1$, and permission probability $r=1$, unless otherwise stated. The load of device $i$ is $\rho_{i}=\lambda W$, where $\lambda$ is the device DAP arrival rate in packets per mini-slot. Then, $\rho_{i}$ is the average number of DAP per data slot that arrive to device $i$. The total traffic load is defined as $\rho_{T}=M \rho_{i}$. Also, $\lambda_{T}=M \lambda$. Three reference traffic loads are considered: i) low, $\rho_{T}=0.3$; ii) medium, $\rho_{T}=0.5$; and iii) high, $\rho_{T}=\{0.8,0.9\}$.

We compare the DAP loss probability and the average DAP delay to validate the analytical and simulation models for systems with $Q=1$. According to (4), the DAP loss probability $P_{L}$ is given by,

$$
P_{L}=1-\frac{\gamma}{M \lambda}=1-\frac{\gamma}{\lambda_{T}} .
$$

Note that for both queue management schemes, TD and PO, the fraction of DAP that are lost must be the same. When a DAP arrives to a full buffer, one DAP is lost regardless of the queue management scheme. With TD, the DAP that arrives is lost, while with PO, the DAP at the head of the queue is lost. This intuition is confirmed by the results of the analytical and simulation models.

Figure 2 shows the variation of the DAP loss probability with the offered load $\rho_{T}$, obtained by the analytical model (ana) and by simulation ( $\operatorname{sim}$ ), for $V=\{2,4\}$. Devices implement the FIFO queue discipline with either tail drop (TD) or push-out (PO). As observed, the analytical and simulation results match exactly.

Note that Fig. 2 displays a somehow counter intuitive system behavior. That is, for loads below $\rho_{T}=0.7, P_{L}$ for a system with $V=2$ is lower than for as system with $V=4$. This is due to the fact that the average frame duration for $V=4$ is longer than for $V=2$. In longer 


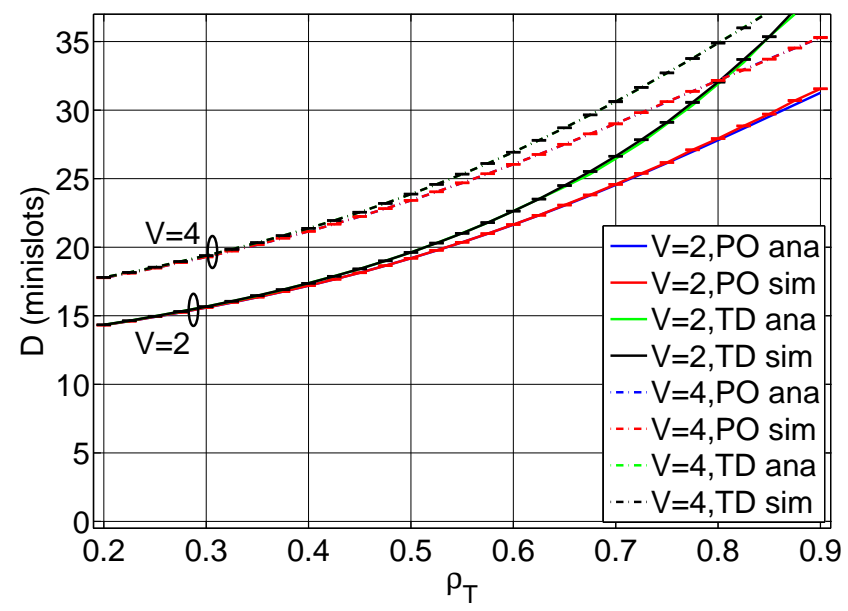

Figure 3: Analytical and simulation average data packet delay with the offered load $\rho_{T}$.

frames, the probability that multiple arrivals occur during the frame is larger. As devices can only hold a single DAP in their buffers, losses will more likely occur in longer frames. However, as load increases, allowing $V=2$ reservation mini-slots instead of $V=4$ becomes a bottleneck. As more collisions occur, DAP are held longer in the buffer, and more losses occur induced by new arrivals. Recall that in FSA-RDP losses may only occur at new DAP arrivals, as collisions in the channel occur with RVP.

Figure 3 shows the variation of the average DAP delay with the offered load $\rho_{T}$, obtained by the analytical model (ana) and by simulation (sim), for $V=\{2,4\}$. The DAP delay is the time elapsed since DAP arrival to departure (when the last bit is transmitted). As observed, the analytical and simulation results for the PO and TD queue management schemes match exactly.

Note in Fig. 3 that the DAP delay for PO is lower than for TD. When PO is implemented and a new DAP arrives to a full buffer, the DAP at the head of the queue is pushed-out. Clearly, pushed-out DAP are the eldest in the queue. This leads to a smaller average DAP delay than with TD, where elder DAP are held, and new ones are lost.

For the numerical results we focus on small values of $M$ and $V$, i.e., clusters with a small number of devices. For large values of $M$ and $V$, the computational complexity of solving the system of linear equations $\boldsymbol{\pi}=\boldsymbol{\pi} \mathbf{P}$ increases substantially. The reader is referred to [48] for the description of standard numerical techniques specific to large systems.

\subsection{Efficiency of the FSA-RDP protocol}

Following with the case $Q=1$, we compare three protocols: i) FSA; ii) FSA-RDP; and iii) ideal FSA-RDP (FSARDP-I). In FSA the contention occurs in data slots (in-slot signaling), while in FSA-RDP occurs in reservation minislots (out-of-slot signaling).

In FSA-RDP-I there is no contention. It is assumed that the controller knows the state of the devices at the
Table 2: Minimum Data Packet Loss Probability $\left(P_{L}\right)$

\begin{tabular}{|c|c|c|c|c|}
\hline$V$ & MAC & $\rho_{T}=0.3$ & $\rho_{T}=0.5$ & $\rho_{T}=0.8$ \\
\hline \multirow{5}{*}{1} & FSA-RDP-I & 0.0109 & 0.0338 & 0.1078 \\
\hline & FSA-RDP & 0.0151 & 0.0484 & 0.1539 \\
\hline & $r_{o p t}$ & 0.64 & 0.52 & 0.39 \\
\hline & FSA & 0.1343 & 0.3097 & 0.5228 \\
\hline & $r_{o p t}$ & 0.38 & 0.29 & 0.21 \\
\hline \multirow{7}{*}{2} & FSA-RDP-I & 0.0115 & 0.0322 & 0.1020 \\
\hline & $\operatorname{sim}$ & 0.0115 & 0.0322 & 0.1021 \\
\hline & FSA-RDP & 0.0131 & 0.0411 & 0.1466 \\
\hline & $\operatorname{sim}$ & 0.0131 & 0.0411 & 0.1466 \\
\hline & $r_{o p t}$ & 1 & 1 & 0.79 \\
\hline & FSA & 0.1243 & 0.3036 & 0.5211 \\
\hline & $r_{o p t}$ & 0.76 & 0.56 & 0.41 \\
\hline \multirow{5}{*}{3} & FSA-RDP-I & 0.0137 & 0.0354 & 0.1040 \\
\hline & FSA-RDP & 0.0154 & 0.0438 & 0.1413 \\
\hline & $r_{o p t}$ & 1 & 1 & 1 \\
\hline & $\mathrm{FSA}$ & 0.1194 & 0.2980 & 0.5196 \\
\hline & $r_{o p t}$ & 1 & 0.82 & 0.61 \\
\hline \multirow{7}{*}{4} & FSA-RDP-I & 0.0162 & 0.0401 & 0.1103 \\
\hline & $\operatorname{sim}$ & 0.0162 & 0.0401 & 0.1103 \\
\hline & FSA-RDP & 0.0179 & 0.0486 & 0.1465 \\
\hline & $\operatorname{sim}$ & 0.0179 & 0.0486 & 0.1465 \\
\hline & $r_{\text {opt }}$ & 1 & 1 & 1 \\
\hline & FSA & 0.1345 & 0.2945 & 0.5184 \\
\hline & $r_{o p t}$ & 1 & 1 & 0.79 \\
\hline \multirow{5}{*}{5} & FSA-RDP-I & 0.0187 & 0.0451 & 0.1186 \\
\hline & FSA-RDP & 0.0205 & 0.0537 & 0.1542 \\
\hline & $r_{o p t}$ & 1 & 1 & 1 \\
\hline & $\mathrm{FSA}$ & 0.1534 & 0.3070 & 0.5173 \\
\hline & $r_{o p t}$ & 1 & 1 & 0.97 \\
\hline \multirow{5}{*}{6} & FSA-RDP-I & 0.0212 & 0.0501 & 0.1277 \\
\hline & FSA-RDP & 0.0231 & 0.0590 & 0.1626 \\
\hline & $r_{o p t}$ & 1 & 1 & 1 \\
\hline & $\mathrm{FSA}$ & 0.1724 & 0.3255 & 0.5198 \\
\hline & $r_{o p t}$ & 1 & 1 & 1 \\
\hline
\end{tabular}

beginning of a frame, and it sends the SAP without the need for contention. In order to perform a fair comparison, the frame structure for FSA-RDP-I is the same as the one defined for FSA-RDP in Fig. 1. That is, it is also composed by the RSF and DSF, and the maximum number of DAP that can be transferred during the DSF is $V$. Therefore, the analytical model for FSA-RDP-I is the same as the one developed for FSA-RDP, except that the permission probability is set to $r=1$, and $D_{k}^{i}(1, V)$ is now defined following (2) as,

$$
D_{k}^{i}(1, V)= \begin{cases}1 & i \leq V, k=i \\ 1 & V<i, k=V \\ 0 & \text { otherwise }\end{cases}
$$

Table 2 shows the minimum $P_{L}$ for different values of $V$ and for the three loads considered. Note that the minimum $P_{L}$ is achieved at the corresponding optimal permission probability $\left(r_{o p t}\right)$, that is obtained by exhaustive search. As expected, the performance of FSA-RDP is upper bounded by the performance of FSA-RDP-I.

Observe that for a given $V, P_{L}$ is larger for FSA than for FSA-RDP. In FSA DAP losses occur mainly due to access collisions, and less frequently due to arrivals to a 
Table 3: Protocol Inefficiency $\left(I_{f}\right)$

\begin{tabular}{llll}
\hline MAC & $\rho_{T}=0.3$ & $\rho_{T}=0.5$ & $\rho_{T}=0.8$ \\
\hline FSA-RDP & 0.20 & 0.27 & 0.39 \\
FSA & 9.99 & 8.14 & 4.08 \\
\hline
\end{tabular}

full buffer. However, in FSA-RDP DAP losses are only due to arrivals to a full buffer.

For a fixed load and a given protocol $X=\{\mathrm{FSA}$, FSA-RDP $\}$, we define $P_{L, X}^{*}$ as the minimum $P_{L}$ obtained from Table 2 for different values of $V$, and denote by $V^{*}$ the value of $V$ at which $P_{L, X}^{*}$ is achieved. For $V<V^{*}$, $P_{L, X}>P_{L, X}^{*}$, as more collisions are expected due to the small number of data slots (FSA) or reservation slots (FSA-RDP). Then, DAP stay longer in the buffer, which increases the probability that new DAP are rejected (TD), or new DAP push-out old DAP from the buffer (PO). Also, for $V>V^{*}, P_{L, X}>P_{L, X}^{*}$, the carried DAP rate does not grow substantially, but frame duration increases (more reservation and data slots). As described before, for $Q=1$ longer frames induce larger DAP losses, as multiple DAP arrivals in a frame more likely occur.

For a given load, we define the inefficiency factor of protocol $X$ as $I_{f, X}=\left(P_{L, X}^{*}-P_{L, I}\right) / P_{L, I}$. Note that $P_{L, I}$ corresponds to the packet loss probability achieved by FSA-RDP-I at the same load and protocol configuration $(V)$ at which $P_{L, X}^{*}$ is found. Clearly, the smaller the value of $I_{f, X}$, the more efficient protocol $X$ is, as its $P_{L, X}$ is closer to the one achieved by the idealized protocol FSA-RDP-I $\left(P_{L, I}\right)$. The inefficiency factors are shown in Table 3. Clearly, the efficiency improvement obtained by FSA-RDP respect to FSA is between one and two orders of magnitude, depending on the load. As observed, the difference between protocol efficiencies decreases as load increases. This effect was expected, as the contention increases with the load.

An interesting observation is that, for a fixed $V$, to achieve the minimum $P_{L}$ in FSA, the optimal permission probability, $r_{\text {opt }}$, has to be appropriately set (values lower than 1), particularly as load increases. However, for FSARDP, the optimal permission probability is constant to 1 for a large range of loads an values of $V$. This would make a scheme that adapts $r$ with the load more robust when implemented with FSA-RDP than with FSA.

\subsection{Packet Delay Distributions}

Figure 4 shows the CDF of data packet delay for three representative offered loads $\rho_{T}=\{0.2,0.5,0.9\}, Q=1$, and for $V=4$. We consider the two queue management schemes, TD and PO. As a reference, we also display the ideal scheduling scheme, denoted by 'I'. For simplicity, we only consider the performance of the POI scheme, that outperforms the TDI scheme.

As expected, observe that DAP delay is smaller for PO than for TD. It has been found that the difference between DAP delays obtained by the PO and TD schemes decreases

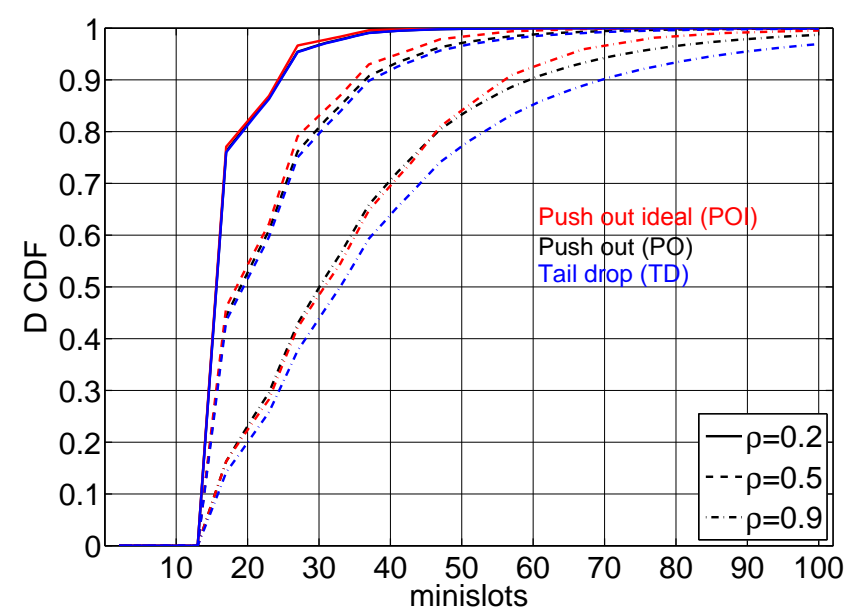

Figure 4: CDF of the data packet delay with the offered load $\rho_{T}$, $V=4$.

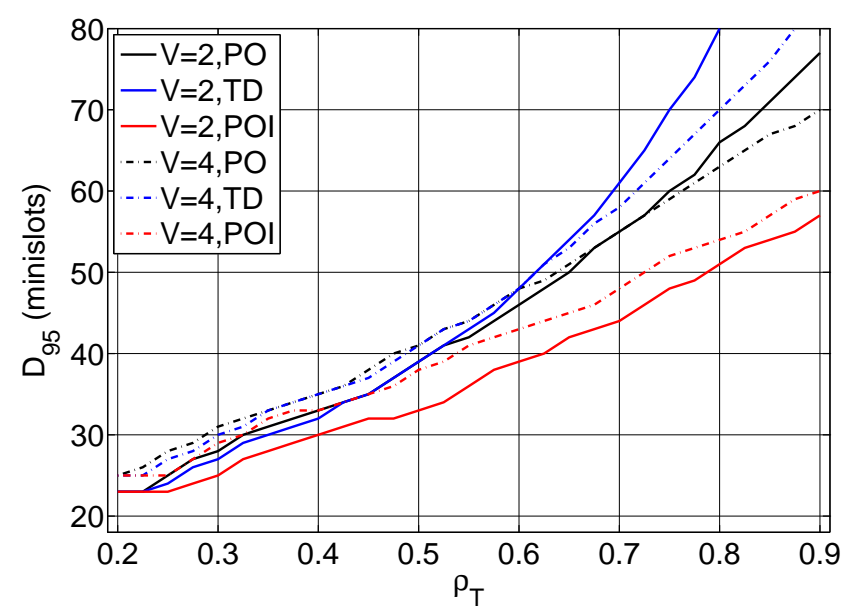

Figure 5: 95th percentile of the data packet delay with the offered load $\rho_{T}$.

as $V$ increases. This is due to the fact that as $V$ increases, a higher reservation success rate is obtained, the devices maintain their queue levels low, and the frequency of DAP push-outs or tail drops decreases.

Figure 5 shows the 95th percentile of the data packet delay, $D_{95}$, with the offered load $\rho_{T}$, for $V=\{2,4\}$. Note that $D_{95}$ is the delay that meets $P\left[D \leq D_{95}\right]=0.95$. That is, $95 \%$ of the transmitted DAP have a delay lower than $D_{95}$. Clearly, for all configurations $D_{95}$ increases with the load. Note that for a constant load and $V$, the $D_{95}$ attained by the TD scheme is larger than the one attained by the PO scheme. Finally, observe that for POI the difference between $D_{95}$ at $V=2$ and at $V=4$ seems to be almost constant with the load.

\subsection{Devices with Larger Queues}

In this section we extend the performance evaluation study to systems with devices that implement buffers of sizes larger than one DAP. This study is made by simulation. More precisely, we now implement devices with 


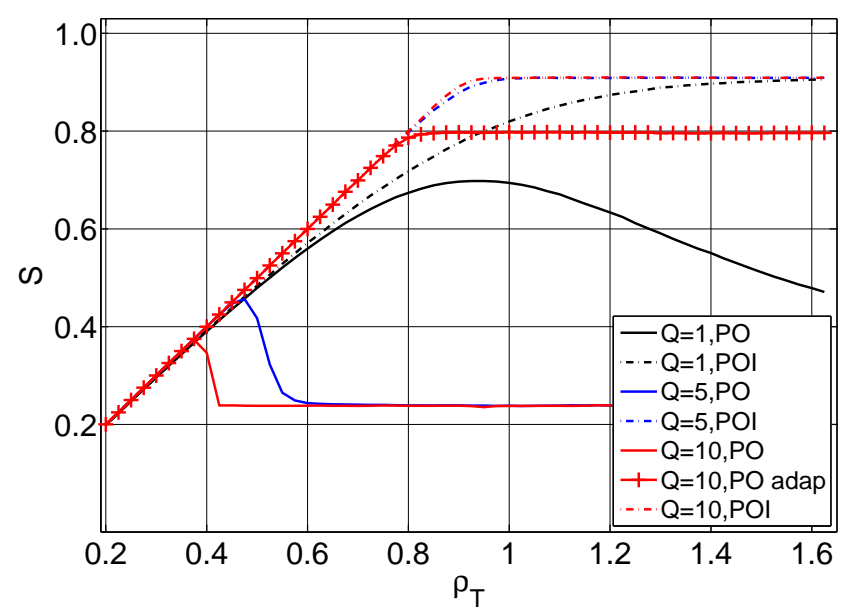

(a) $V=2$

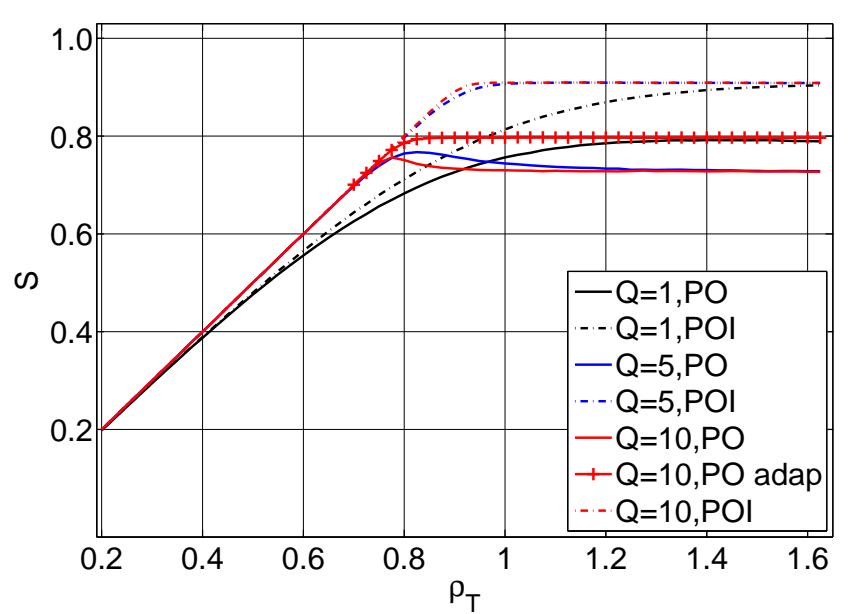

(b) $V=4$

Figure 6: Channel utilization with the offered load $\rho_{T}$.

two queue sizes, $Q=\{5,10\}$ DAP. We study two scenarios where the number of reservation mini-slots per RSF are $V=\{2,4\}$, and the permission probability is always set to $r=1$, unless otherwise stated. Here, we also have considered FIFO as the service discipline and two queue management schemes, TD and PO.

Figure 6 shows the evolution of the channel utilization $S$ with the load for systems with $V=2,4$. It is defined as,

$$
S=W \gamma
$$

and expresses the fraction of time the channel is being occupied with DAP, i.e., RVP are considered control overhead. Although figures show results for the PO scheme, they coincide with the ones obtained for the TD scheme.

For $Q=\{1,5,10\}$, and $V=2$, the maximum achieved by FRA-RDP-I is $S_{\max }=0.908$. For $V=4$ the curves are identical to the ones shown for $V=2$. This similarity is expected. We consider that the FRA-RDP-I scheme operates in the saturation regime when all nodes are active in all frames. Then, at each frame the number of DAP transmitted will be $V$ (provided that $M \geq V$ ). Then, the channel utilization achieved by the FRA-RDP-I scheme in the saturation regime is given by,

$$
S_{s}=\frac{V W}{V+V W}=\frac{W}{1+W},
$$

i.e., $S_{s}$ is independent of $V$ and only depends on $W$. For $W=10, M=8$ and $V=2$ or $V=4$, we get $S_{s}=10 / 11=$ $0 . \overline{90}$, that approximately coincides with the maximum for FRA-RDP-I obtained by simulation and provided above. This explains why the curve of $S$ for $V=2$ coincide with the one for $V=4$ when FRA-RDP-I is used.

For $Q=1, V=2, \mathrm{FRA}-\mathrm{RDP}$ peaks at $S_{\max }=0.698$. For $V=2$, we observe that $S$ collapses for systems with $Q=5$ for loads $\rho_{T}>0.47$, and for systems with $Q=10$ for loads $\rho_{T}>0.40$. This phenomenon is due to the fact that, as load increases, devices get to a point where they stay active (with DAP in their queues) during all frames. That is, all devices contend for reservation slots in all frames. This leads to massive collisions, throughput reduction and massive losses.

This intuition can be validated using the analytical model and assuming that, in the saturation regime of the FRA-RDP scheme, $\pi_{M}=1$ and $\pi_{i}=0, i \neq M$. As an example, for $V=2, r=1$, the throughput in saturation (collapsed regime) can be computed from (4) simply by,

$$
\gamma_{s}=\sum_{k=0}^{V} k f_{k} / \sum_{k=0}^{V} t_{k} f_{k}, \quad f_{k}=D_{k}^{M}(r=1, V),
$$

where $D_{k}^{M}(r=1, V)$ is the probability of $k$ success reservations when $M$ devices contend, and was given by (2). Clearly, $S_{s}=W \gamma_{s}$. Then we get, $S_{s}(M=8, V=2)=$, 0.238 , that coincides with the simulation value represented in Fig. 6a.

For $V=4$, FRA-RDP achieves $S_{\max }=0.767$ when $Q=5$, and $S_{\max }=0.756$ when $Q=10$. In saturation they tend to $S_{s}=0.721$. This is validated by computing analytically $S_{s}(M=8, V=4)$ using (32).

Figure 7 shows the evolution of the channel utilization in the saturation regime $S_{s}$ with the number of devices in the network $M$, when $r=1$. The curve for $V=M$ displays $S_{s}$ for the optimal configuration of the RSF, i.e, the number of reservation mini-slots equals the number of contenders per frame. Recall that in the saturation regime all devices contend in all frames. Curves for $V=M-1$ and $V=M+1$ display $S_{s}$ for suboptimal configurations of the RSF. These two curves are important as they show that as $M$ increases, the optimal configuration of the number of reservation mini-slots in the RSF becomes less critical. That is, the system performance achieved by an adaptive scheme that would configure $V$ as a function of the load would be almost insensitive to small errors in the estimation of $M$. 


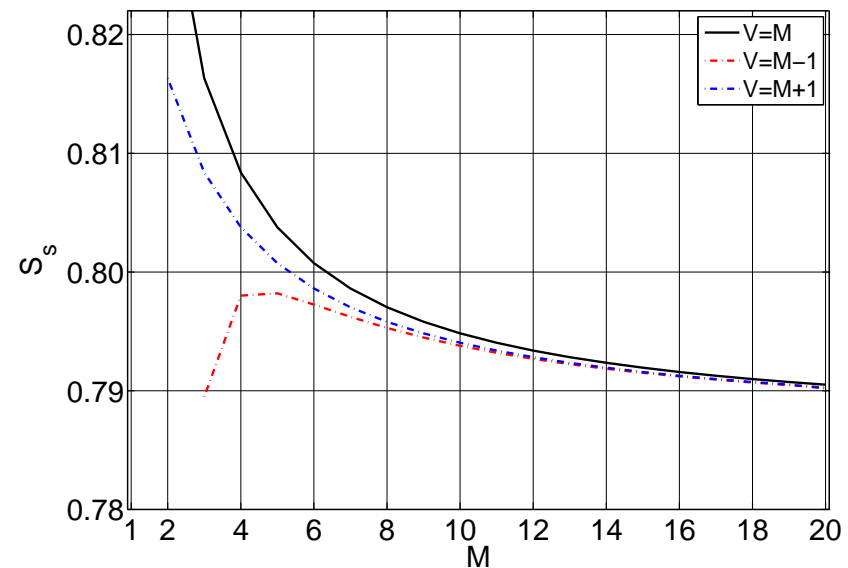

Figure 7: Channel utilization as a function of the number of devices in the saturation regime.

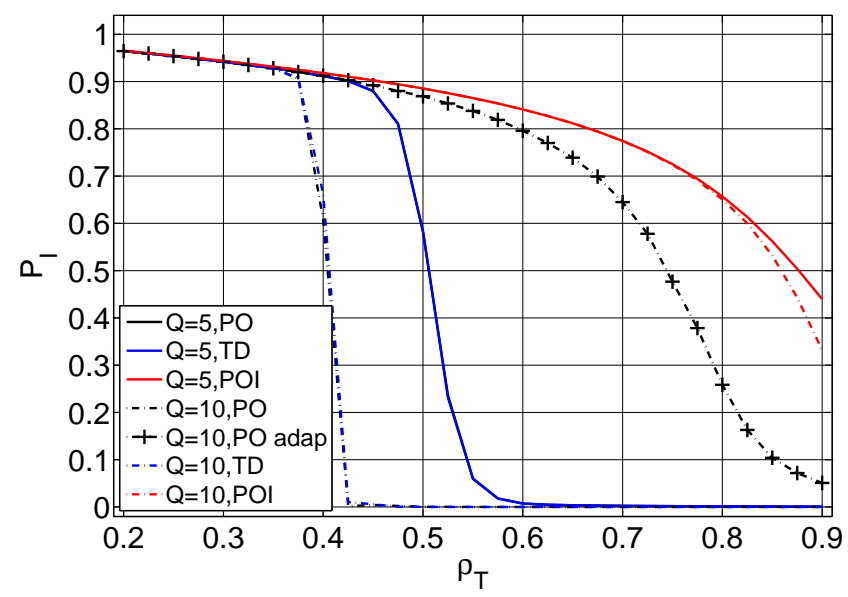

Figure 8: Fraction of frames a device is inactive with the offered load $\rho_{T}, V=2$.

Figure 8 shows the evolution of the fraction of frames a device is inactive (empty queue) for a system with $V=2$. As observed, when load grows above the values that lead to a system collapse in Fig. 6a, the corresponding devices become always active $\left(P_{I} \approx 0\right)$, and therefore, all devices contend in all frames. This result helps to understand why the system with $Q=10$ collapses earlier (smaller load) than with $Q=5$.

Figures 9, 10 and 11 show the evolution of the DAP loss probability $P_{L}$, the average DAP delay $D$, and the 95 th percentile of the DAP delay $D_{95}$ with the total offered load $\rho_{T}$. The shape of the curves in these figures provide additional evidences of the system collapse phenomenon described previously. Note that the DAP loss probability $\left(P_{L}\right)$ curves for PO and TD coincide. For the average DAP delay $D$, both schemes achieve similar performance at low loads. However, for high loads, the PO scheme achieves a slightly lower average DAP delay than the TD scheme for the same load. This difference was explained before.

Observe that, the evolution of $D_{95}$ with the load has a characteristic knee shape. The knee occurs at approxi-
Table 4: Representative values for the adaptive scheme

\begin{tabular}{cccccccc}
\hline$\rho_{T}$ & 0.2 & 0.4 & 0.6 & 0.7 & 0.75 & 0.8 & 0.9 \\
\hline$V$ & 2 & 2 & 2 & 2 & 3 & 3 & 3 \\
$r$ & 1.00 & 0.95 & 0.65 & 0.55 & 0.50 & 0.50 & 0.40 \\
\hline
\end{tabular}

mately the same load for both the PO and the TD schemes, with negligible differences. Although not observable in Fig. 11, $D_{95}$ for the PO scheme is slightly lower than the one for the TD scheme. The knee point occurs after the curves for $P_{L}$ start to grow exponentially. In the proximity of the knee, all devices are active in all frames, the throughput is very small as a lot of RVP collisions occur, and DAP stay longer in the queue.

A final observation is that as $V$ increases, the difference between the performance of FSA-RDP with $Q=5$ and $Q=10$ is drastically reduced, and they approach the performance of the FSA-RDP-I scheme. Note also that the performance of the FSA-RDP-I scheme for $V=2$ and $V=4$ is quite similar. This is due to the fact that the DAP loss probabilities experienced by the ideal scheme for $V=2$ and $V=4$ are quite similar (in fact negligible). This is an important observation, as it shows that their is still room for improvement in the design of new scheduling schemes that use more efficiently the system resources, particularly for $V<M$.

\subsection{Adaptive Scheme}

One possible solution to the collapse condition problem displayed in Fig. 6a would be to implement an adaptive scheme. It should adjusts both the number of reservation slots $V$ and the permission probability $r$ as a function of the load. Note that the system load varies with the product $M \lambda$.

To validate this intuition we provide results for a system where devices have a buffer of $Q=10 \mathrm{DAP}$. In this system, the controller adapts $V$ and $r$, and informs the devices by using, for example, the slot allocation packet (SAP) at the end of the RSF, or the ACK packet at the end of the DSF. The adaptation algorithm at the controller might be based on the number of successes and collisions observed during past RSF. When device $i$ is active, it contends for one of the $V$ reservation mini-slots with probability $r$, where the values of $V$ and $r$ have been broadcast by the controller. With probability $1-r$, it sleeps until the next frame.

Table 4 shows some representative values for the adjusted $V$ and $r$ found by exhaustive search, when the initial value is $V=2$ and for the load range $\rho_{T} \in[0.2,0.9]$. Observe that at $\rho_{T}=0.75, V$ is increased by one unit.

The maximum channel utilization $S_{\max }$ can also be improved by adapting $V$ and $r$ as a function of the load. For example, Fig. 6a displays the result of adapting the system with $Q=10$ in the interval $\rho_{T} \in[0.2,1.6]$ (curve 'PO adap'). $S_{s}$ improves from 0.238 when a static configuration $V=2, r=1$, is used, to 0.8 when adaptation is used. Also, Fig. 6b displays the result of adapting the 


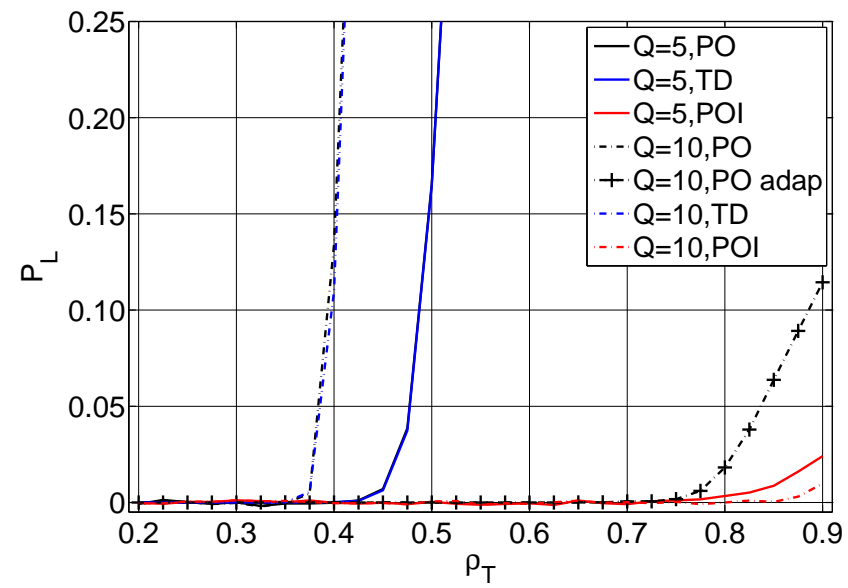

Figure 9: Data packet loss probability with the offered load $\rho_{T}$, $V=2$.

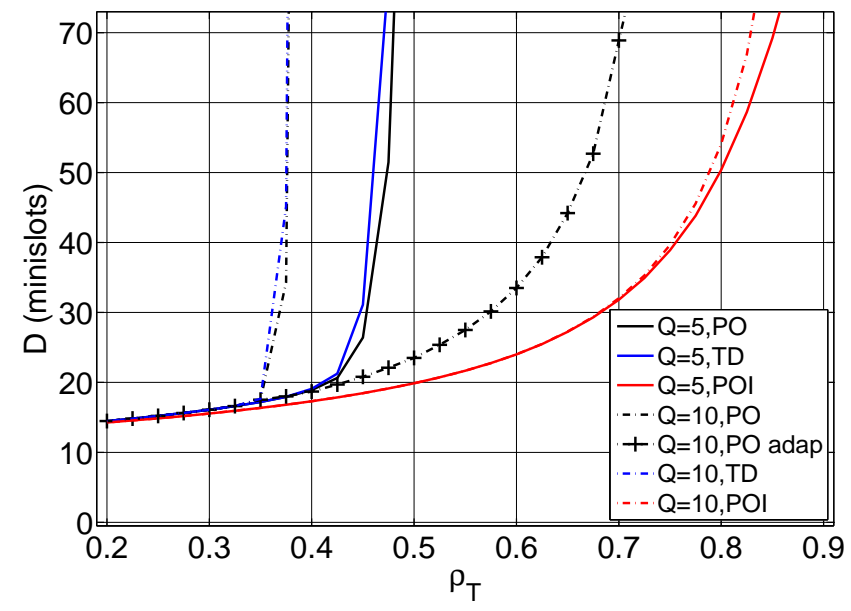

Figure 10: Average data packet delay with the offered load $\rho_{T}, V=$ 2.

system with $Q=10$ in the interval $\rho_{T} \in[0.7,1.6]$ (curve 'PO adap'). $S_{s}$ improves from 0.721 when a static configuration $V=4, r=1$, is used, to 0.8 when adaptation is used. Note that as the load increases $\left(\rho_{T}>0.9\right), V$ must be increased progressively up to $V=8$.

Figures 9, 10, and 11, also show the impact that the adaptation of $V$ and $r$ have on the system performance. As observed, the system performance improves drastically, as $P_{L}, D, D_{95}$ all decrease. Clearly, adaptation allows the system to successfully operate over a wider load range than without adaptation.

For a scenario with $V=4$ (not shown), when $Q=5$ the average delay $D$ for the $\mathrm{PO}$ and TD schemes are identical. The same happens when $Q=10$. The average delay $D$ obtained when $Q=5$ and $Q=10$ are very close to the one achieved by the adaptive scheme in the scenario with $Q=10$ and $V=2$. That is, as expected, for a constant load, the adaptation becomes less relevant as $V$ increases. A similar comment can be said for $D_{95}$.

For the DAP loss probability $P_{L}$ in a scenario with $V=$ 4 (not shown), a similar comment can be said. However,

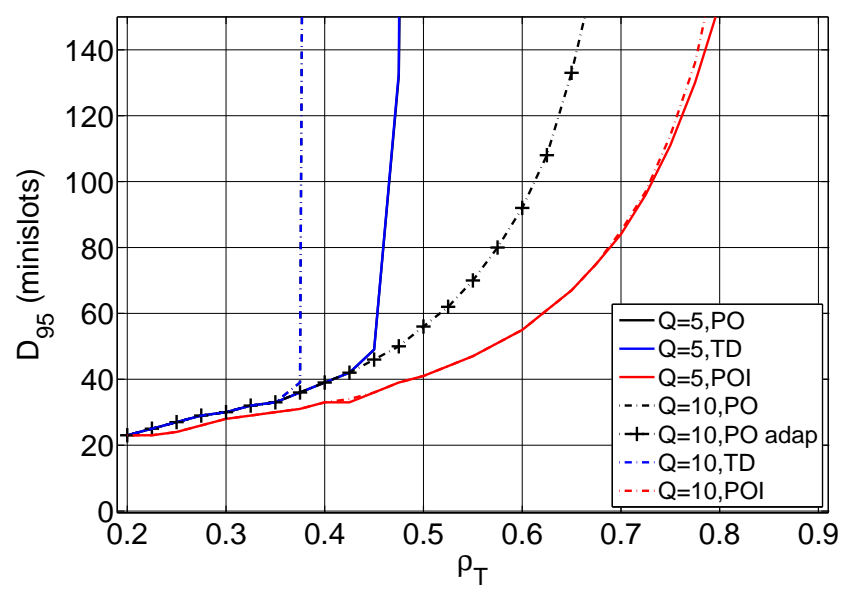

Figure 11: 95th percentile of the data packet delay with the offered load $\rho_{T}, V=2$.

the $P_{L}$ achieved in scenarios with $Q=5$ and $Q=10$ is slightly worse than the one achieved by the adaptive scheme in the scenario with $Q=10$ and $V=2$. Figure 8 shows the beneficial impact that adaptation has on $P_{I}$.

From the energy efficiency point of view, it is desirable to achieve an energy consumption per successfully transmitted bit as low as possible. Then, collisions in the RSF should be minimized. Focusing on the system with $Q=10$ as an example, it is clear that when the load goes beyond the collapse point, the system remains in a state where the energy is wasted by the transmission of RVP that collide, and a very low throughput is obtained, achieving a poor energy efficiency. Then, an adaptive scheme would drastically help to reduce energy consumption, and improve energy efficiency.

\subsection{Successive Interference Cancellation}

We study two scenarios where devices implement FSA$\mathrm{RDP}$, and in addition the controller implements successive interference cancellation (SIC) techniques to achieve multipacket detection. In the first scenario devices implement Irregular Repetition Slotted ALOHA (IRSA) [25], and we assume a perfect channel. In the second scenario we assume an imperfect channel, but devices transmit a single RVP per frame. That is, no RVP replicas are transmitted in the second scenario.

\subsubsection{IRSA}

When nodes implement IRSA, they transmit a random number of RVP replicas per frame. We evaluate the performance of IRSA in scenarios with pairs $(M, V)=$ $\{(20,20),(200,200)\}$. The permission probability is always set to $r=1.0$. Devices operate in saturation mode, and transmit one mandatory RVP per RSF. Further RVP can be transmitted following a truncated geometric distribution with probability $q$, up to a maximum of $V$ RVP. Let $R_{p}$ be the number of RVP that a device transmits in the same frame. Then, $P\left[R_{p}=n\right]=q^{n-1}(1-q), 1 \leq n \leq$ $V-1$, and $P\left[R_{p}=V\right]=q^{V-1}$. The average number of 
Table 5: IRSA, $M=20, V=20$

\begin{tabular}{ccccccc}
\hline$q$ & $\overline{R_{p}}$ & $R_{S}$ & $S$ & $\Delta_{S}$ & $E E$ & $\Delta_{E E}$ \\
\hline 0 & 1.00 & 7.54 & 0.79 & & $79.04 \%$ & \\
0.1 & 1.11 & 7.88 & 0.80 & $0.91 \%$ & $78.00 \%$ & $-1.31 \%$ \\
0.2 & 1.25 & 8.22 & 0.80 & $1.76 \%$ & $76.68 \%$ & $-2.98 \%$ \\
0.3 & 1.43 & 8.63 & 0.81 & $2.71 \%$ & $75.12 \%$ & $-4.96 \%$ \\
0.4 & 1.67 & 9.05 & 0.82 & $3.62 \%$ & $73.08 \%$ & $-7.54 \%$ \\
0.5 & 2.00 & 9.27 & 0.82 & $4.07 \%$ & $69.86 \%$ & $-11.62 \%$ \\
0.6 & 2.50 & 8.59 & 0.81 & $2.64 \%$ & $63.22 \%$ & $-20.01 \%$ \\
0.7 & 3.33 & 5.45 & 0.73 & $-7.43 \%$ & $45.02 \%$ & $-43.04 \%$ \\
0.8 & 4.94 & 1.02 & 0.34 & $-57.23 \%$ & $9.37 \%$ & $-88.15 \%$ \\
0.9 & 8.78 & 0.004 & 0.002 & $-99.75 \%$ & $0.02 \%$ & $-99.97 \%$ \\
\hline
\end{tabular}

Table 6: IRSA, $M=200, V=200$

\begin{tabular}{ccccccc}
\hline$q$ & $\overline{R_{p}}$ & $R_{S}$ & $S$ & $\Delta_{S}$ & $E E$ & $\Delta_{E E}$ \\
\hline 0 & 1.00 & 73.76 & 0.79 & & $78.67 \%$ & \\
0.1 & 1.11 & 76.71 & 0.79 & $0.83 \%$ & $77.54 \%$ & $-1.90 \%$ \\
0.2 & 1.25 & 80.12 & 0.80 & $1.72 \%$ & $76.22 \%$ & $-3.57 \%$ \\
0.3 & 1.43 & 84.11 & 0.81 & $2.70 \%$ & $74.64 \%$ & $-5.56 \%$ \\
0.4 & 1.67 & 88.97 & 0.82 & $3.79 \%$ & $72.75 \%$ & $-7.96 \%$ \\
0.5 & 2.00 & 95.20 & 0.83 & $5.05 \%$ & $70.41 \%$ & $-10.91 \%$ \\
0.6 & 2.50 & 102.74 & 0.84 & $6.40 \%$ & $67.27 \%$ & $-14.89 \%$ \\
0.7 & 3.33 & 86.18 & 0.81 & $3.17 \%$ & $56.38 \%$ & $-28.66 \%$ \\
0.8 & 5.00 & 9.41 & 0.32 & $-59.33 \%$ & $8.60 \%$ & $-89.12 \%$ \\
0.9 & 10.00 & 0.07 & 0.004 & $-99.53 \%$ & $0.04 \%$ & $-99.95 \%$ \\
\hline
\end{tabular}

RVP sent per frame is given by, $\overline{R_{p}}=\left(1-q^{V}\right) /(1-q)$. Each RVP transmitted by a device selects one reservation mini-slot randomly, avoiding multiple selections of the same mini-slot in the same RSF.

Tables 5 and 6 show the average number of successful RVP detections at the receiver per frame $R_{S}$. A RVP is detected successfully when it is the only RVP in a reservation mini-slot. In addition, when a RVP is detected using the SIC technique, its signal is subtracted from the signal of those mini-slots containing replicas of the successfully detected RVP. Then, new mini-slots with a single RVP might appear, and can now be successfully detected. The procedure continues until no more RVP can be detected.

Columns labeled with $S$ show the achieved throughput defined as,

$$
S=\frac{W R_{S}}{V+W R_{S}},
$$

where $W$ is the number of mini-slots occupied by a DAP. Columns labeled with $\Delta_{S}$ show the relative throughput improvement compared to a conventional system that does not implement IRSA $(q=0)$. Clearly, $R_{S}$ and $S$ increases with $q$ up to a given value, and then sharply decrease.

Columns labeled with $E E$ show the energy efficiency defined as,

$$
E E=\frac{W R_{S}}{M \overline{R_{p}}+W R_{S}},
$$

where $M \overline{R_{p}}$ is the energy spent to transmit RVP, and $W R_{S}$ the energy spent to transmit DAP, both per frame. Clearly, we assume equal transmission power for RVP and
DAP. Recall that the duration of a RVP is one mini-slot. Columns labeled with $\Delta_{E E}$ show the relative energy efficiency improvement compared to the one achieved by a system that does not implement IRSA $(q=0)$.

From the results summarized in Tables 5 and 6 we conclude that IRSA does not seem to be a convenient scheme for wireless sensor networks, where devices are battery limited. As observed, for small systems $((M, V)=(20,20))$, to achieve a throughput improvement of $4 \%$, the energy efficiency decreases by $12 \%$, approximately. For larger systems $((M, V)=(200,200))$, to achieve a throughput improvement of almost $6 \%$, the energy efficiency decreases by $15 \%$, approximately.

\subsubsection{No Reservation Packet Replicas}

The impact of propagation in the channel is commonly described by three effects: path loss, shadowing and fast fading [49]. For the path loss we follow the IEEE channel model $[50,51]$. For additional details on the channel model and the parameters used, please refer to [52]. To be consistent with the analytical model that considers that all active devices have the same success probability when they contend for the channel, we assume that all devices are at the same distance $(d)$ from the controller.

A channel model that takes into account only the shadowing effect, but not the fast fading, is more realistic for wireless sensor scenarios, where nodes are mostly static. The results in [52] show that the average number of successfully decoded RVP in the same reservation mini-slot when the channel model only takes into account shadowing is close to the one obtained when the channel model takes into account both shadowing and fast fading. Then, for the rest of the study we assume a channel model that only considers the shadowing (slow fading) effect. Results also show that SIC provides an important performance boost, as it will shown later. As expected, the performance of SIC deteriorates as $d$ increases, or the number of RVP in the same mini-slot increases.

In the scenarios studied, curves not labeled with 'SIC' refer to scenarios where a perfect channel is assumed, and all transmitted RVP are received successfully, provided they do not collide. However, in SIC scenarios an imperfect (lossy) channel is assumed, and not all transmitted RVP might be successfully received. In scenarios with imperfect channel, for simplicity, we assume that channel impairments affect only to RVP, while data packets are always successfully received. There, we study three representative distances: $100 \mathrm{~m}$ (short), $600 \mathrm{~m}$ (medium) and $1000 \mathrm{~m}$ (long). As the results obtained for medium distances are quite close to the ones obtained for short distances, medium distance results are not shown for brevity.

Figure 12 shows the impact of implementing SIC in a system where devices have a limited buffer of size $Q=1$ DAP. We represent the cumulative distribution function of the delay of DAP that are successfully transmitted $(D)$ 


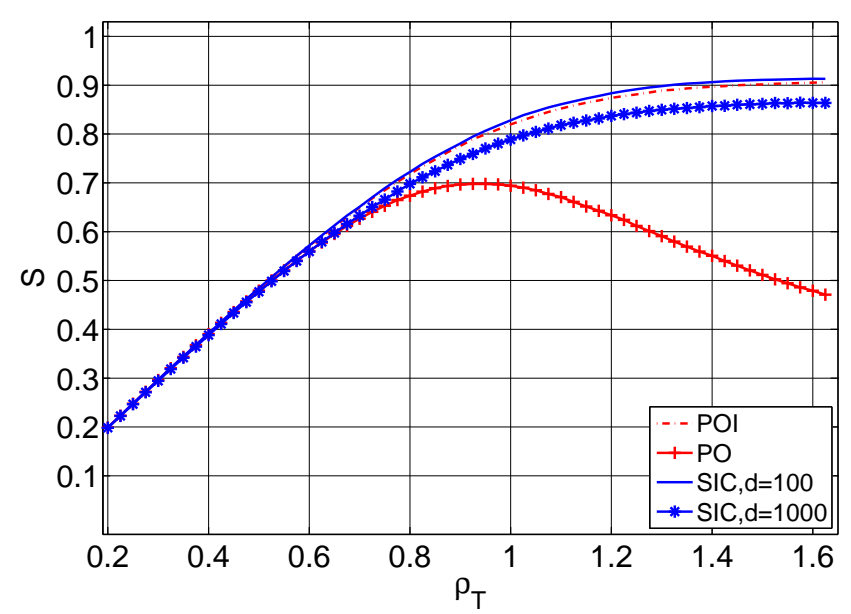

(a) $Q=1, V=2$

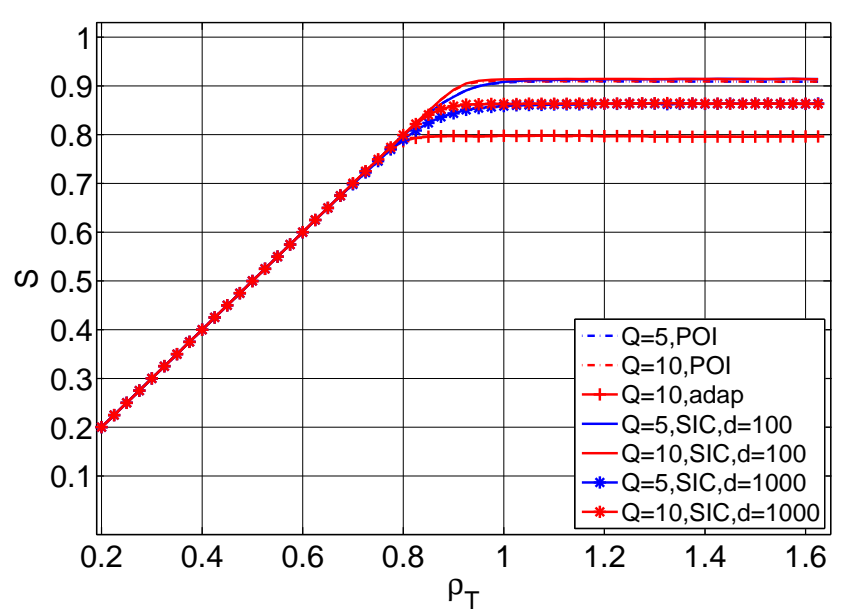

(b) $V=4$

Figure 13: Channel utilization with the offered load $\rho_{T}$.

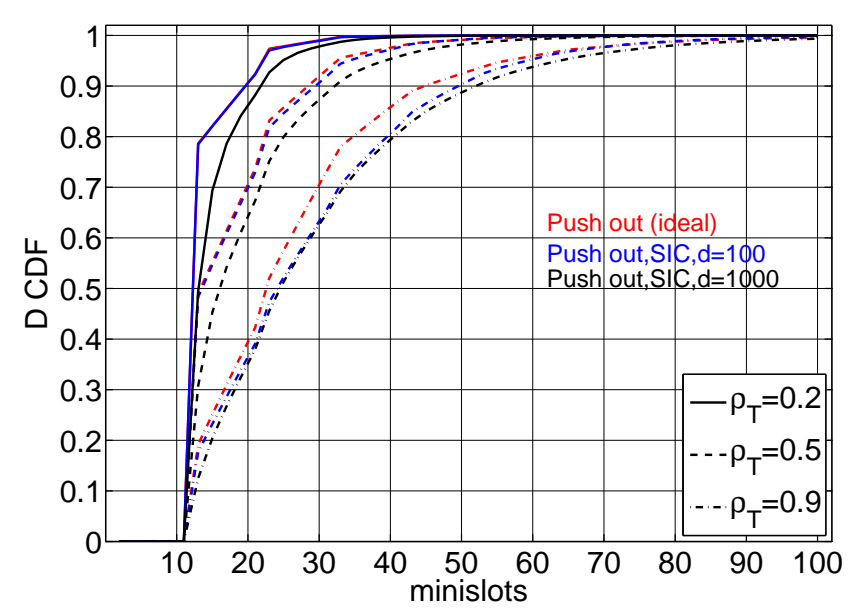

Figure 12: CDF of the data packet delay for different offered loads $\rho_{T}$, and $V=2$.

when $V=2$. The push-out ideal (POI) discipline describes an idealized operation that was described in Subsection 6.1. Clearly, for short to medium distances (100$600 \mathrm{~m}$ ), the performance of a system operating with SIC is almost identical to the performance of the POI scheme.

Figure 13 shows the evolution of the system throughput $S$ with the offered load $\rho_{T}$. Observe that in the scenarios shown, the SIC scheme in short to moderate distances achieves a slightly higher throughput than the POI scheme. This is an outstanding result, as it shows the huge potential of SIC techniques. The advantage of implementing SIC comes from the fact that with a frame of $V$ RSV slots, a maximum of $V$ DAP per frame can be transmitted when the POI scheme is used. However, when SIC techniques are used at the receiver (controller) more that $V$ RVP per RSF might be successfully received, and therefore more that $V$ DAP per frame can be transmitted.

An additional interesting observation is that even in the worst scenario with $d=1000 \mathrm{~m}$, the SIC scheme out-

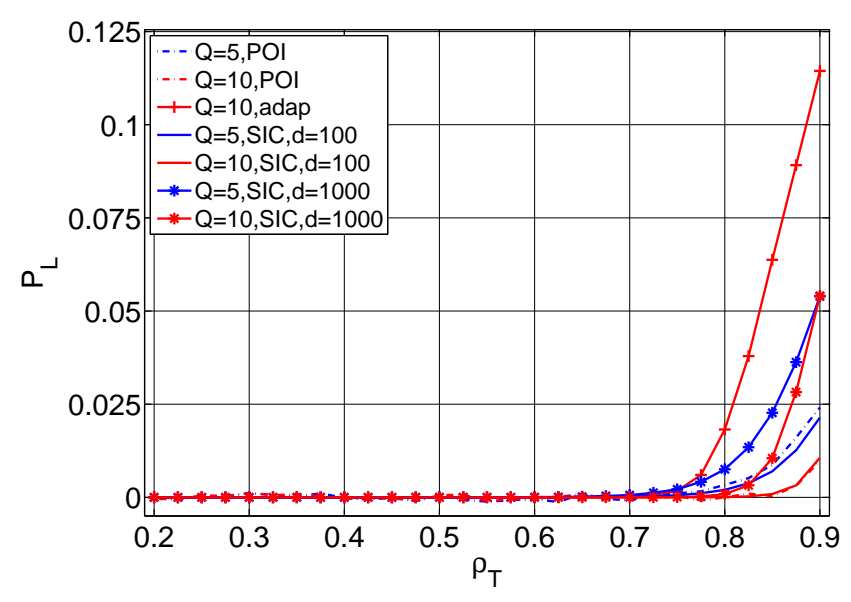

Figure 14: Data packet loss probability with offered load $\rho_{T}, V=2$.

performs the adaptive scheme. Curves with ' $\mathrm{Q}=10$,adapt' refer to the performance of a system that assumes a perfect channel, uses a push-out queue management scheme, and adapts the permission probability $r$ and the number of reservation mini-slots $V$. The adaptive scheme was described in Subsection 6.4.

Figures 14 and 15 confirm the performance improvement brought by SIC. Note that, as in previous sections, packet losses in Fig. 14 are only due to DAP arrivals to a full buffer and not to channel impairments. As observed before, even in the worst scenario $(d=1000)$, the performance of a SIC scheme is better that the one obtained by the adaptive scheme. The only exception is for the delay performance parameters $D$ (not shown) and $D_{95}$ at $d=1000 \mathrm{~m}$, where the adaptive scheme (perfect channel) obtains a small advantage at low loads when compared to the performance of SIC [52]. This is due to the fact that as distance increases, the SINR of the received RVP decreases and the detection performance of the SIC scheme deteriorates. Then, DAP stay longer in the queue. 


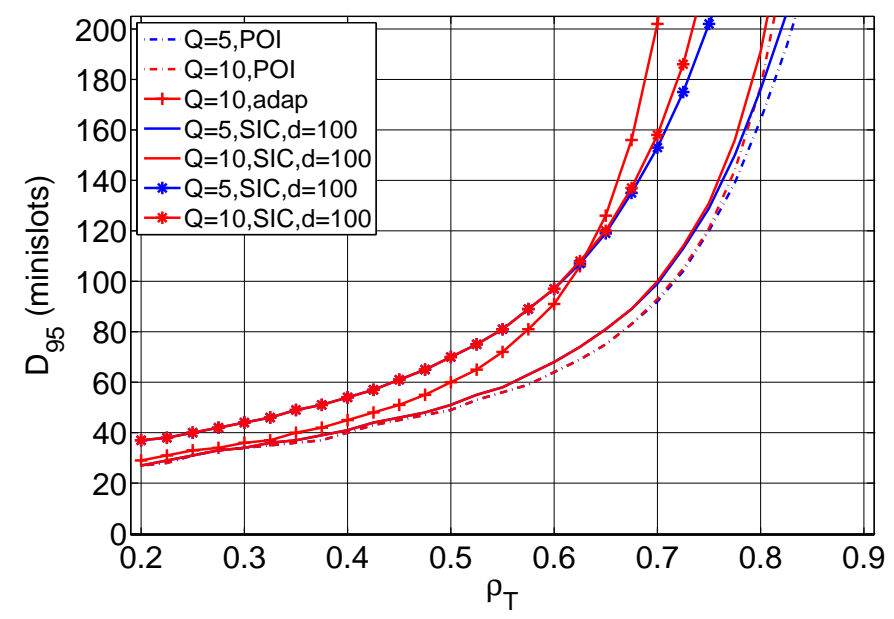

Figure 15: 95th percentile of the data packet delay with the offered load $\rho_{T}, V=4$.

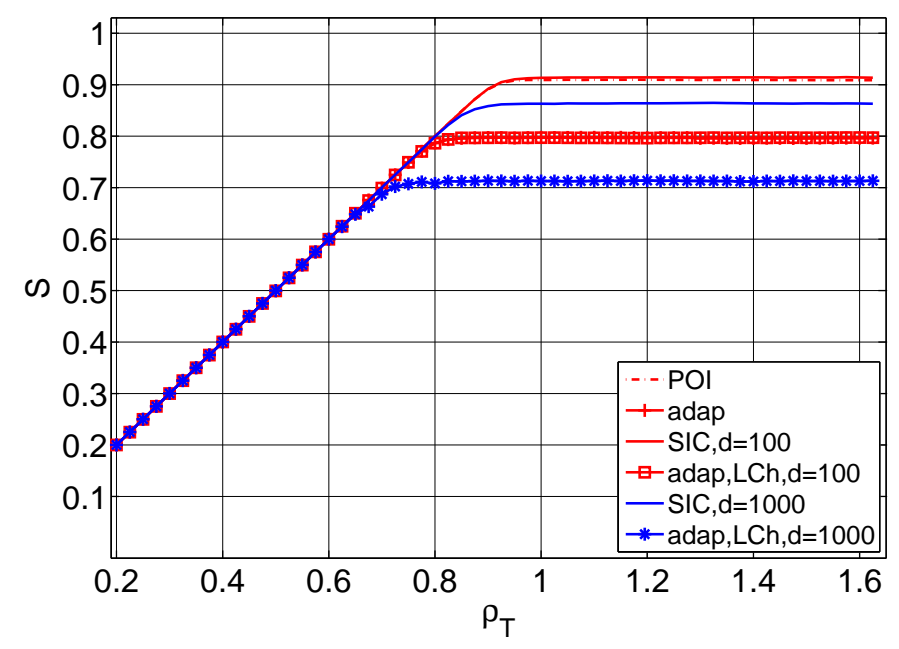

Figure 16: Channel utilization with the offered load $\rho_{T}, V=4$, $Q=10$ adaptive.

Figure 16 shows the evolution of the system throughput with the offered load in scenarios where devices have queues of size $Q=10$ DAP. We study two scenarios, one that assumes perfect channel and one that assumes an imperfect (lossy) channel (LCh). In scenarios with an imperfect channel, we assume that the detection hardware of the controller operates with or without SIC. In some scenarios the controller adapts the access permission probability $r$ and the number of reservation mini-slots $V$. In scenarios with an imperfect channel, we have reused optimal values of $r$ and $V$ obtained for perfect channel. Note in Fig. 16 that the reused values are not the optimal ones.

The main purpose of curves 'adap,LCh, $\mathrm{d}=100$ ' and 'adap,LCh, $d=1000$ ' is to show the performance of the adaptive scheme in a more realistic scenario (lossy channel). This provides a fairer performance comparison with the SIC scheme. As expected, the difference between the throughput achieved by the adaptive scheme in a scenario with perfect channel and with imperfect channel is negli- gible at short distances $(d=100)$. Also, the throughput achieved by the adaptive scheme and by the SIC scheme is practically the same for small loads. However, at medium to high loads, the throughput achieved by the SIC scheme is noticeably higher. At long distances $(d=1000)$ the relative throughput loss of the adaptive scheme is larger than the relative throughput loss of the SIC scheme, when compared to the ones achieved at short distances $(d=100)$. Table 7 shows the energy efficiency of a system in different evaluation scenarios. Columns labeled with ' $\mathrm{ICh}$ ' refer to scenarios where a perfect (ideal) channel and no SIC at the controller is assumed. Columns labeled with ' $\mathrm{LCh}$ ' refer to scenarios where an imperfect (lossy) channel and no $\mathrm{SIC}$ at the controller is assumed. Finally, columns labeled with 'SIC' refer to scenarios where an imperfect channel and SIC at the controller is assumed. Scenario labeled with $Q=10^{*}$ refer to a system that adapts the permission probability and the number of reservation mini-slots. Energy efficiency is determined by (34), but now no RVP replicas are sent.

For imperfect channels, high load and $V=2$ (not shown), the adaptive scheme achieves an energy efficiency higher than the one obtained by the SIC scheme [52]. However, for $V=4$ the SIC scheme achieves a slightly better energy efficiency. This suggests that in some scenarios where the controller implements SIC, the energy efficiency might be further improved by adopting the adaptive scheme. Alternatively, when the additional cost of implementing SIC is not justified, the performance and energy efficiency of the system can still be quite good by implementing the adaptive scheme, particularly for low loads and short to medium distances.

\section{Conclusions}

In this paper we studied a type of Frame Slotted ALOHA protocol that proposes reservation and data packets, that we refer to as FSA-RDP. In FSA-RDP frames are divided in two subframes, the reservation and the data subframes. In reservation subframes active devices that have access permission contend for sending reservation packets to the controller. Those that succeed (their reservation packets do not collide) are assigned a dedicated data slot to transfer a DAP during the data subframe.

We model the operation of the FSA-RDP protocol with a discrete-time Markov chain, and determine the protocol efficiency and the packet delay distribution, for a scenario where M2M devices have a buffer to store a single packet. We define the protocol efficiency in terms of the data packet loss probability. We compare the efficiency of the FSA-RDP protocol and the conventional Frame Slotted ALOHA (FSA). Results show that protocol efficiency of FSA-RDP is between one and two orders of magnitude larger than the efficiency of the conventional FSA.

We determine the cumulative distribution function of the packet delay for FSA-RDP, when both the tail drop (TD) and push-out (PO) queue management schemes are 
Table 7: Energy Efficiency, $V=4$

\begin{tabular}{|c|c|c|c|c|c|c|c|c|}
\hline \multirow[b]{3}{*}{$\rho_{T}$} & \multicolumn{4}{|c|}{$Q=10$} & \multicolumn{4}{|c|}{$Q=10^{*}$} \\
\hline & \multirow[t]{2}{*}{$\mathrm{ICh}$} & \multicolumn{3}{|c|}{$\mathrm{SIC}$ (distance, $d$ ) } & \multirow[t]{2}{*}{$\mathrm{ICh}$} & \multicolumn{3}{|c|}{$\mathrm{LCh}$ (distance, $d$ ) } \\
\hline & & 100 & 600 & 1000 & & 100 & 600 & 1000 \\
\hline 0.200 & $90.55 \%$ & $90.84 \%$ & $89.60 \%$ & $86.16 \%$ & $90.55 \%$ & $90.55 \%$ & $89.28 \%$ & $85.64 \%$ \\
\hline 0.800 & $63.65 \%$ & $88.97 \%$ & $87.00 \%$ & $81.62 \%$ & $80.15 \%$ & $80.15 \%$ & $75.92 \%$ & $66.69 \%$ \\
\hline 1.625 & $57.17 \%$ & $84.20 \%$ & $81.57 \%$ & $76.03 \%$ & $82.71 \%$ & $79.70 \%$ & $77.40 \%$ & $71.28 \%$ \\
\hline
\end{tabular}

deployed. We study different loads and configurations for the protocol. Results show that the difference between the delay obtained by both queue management schemes is only significant for high loads, and when the number of mini-slots in the reservation subframe is small, i.e., when the collision rate is high.

We also studied the system performance with buffer sizes of 5 and 10 packets. We observed that, as the load increases, the system collapses, bringing the throughput to very low values and, in turn, the packet loss probability and packet delay to a very high ones. An adaptive scheme that adjust the number of reservation slots $V$ and the permission probability $r$ at every frame is suggested to cope with this problem. Results show a substantial performance improvement when adaptation is performed.

Finally, we studied the impact that implementing Successive Interference Cancellation (SIC) at the controller has on the system performance. We evaluated the performance of implementing SIC at the controller together with Irregular Repetition Slotted ALOHA (IRSA) to send the reservation packets, assuming a perfect channel. When compared to a conventional system that does not use packet replicas, IRSA achieves a rather modest throughput improvement, but at the expense of a higher energy consumption. We also analyzed scenarios with imperfect channel, where the controller implements SIC. We compared the performance of systems that implement SIC to those that do not implement SIC. In these more realistic channel environments, we showed that the use of SIC brings an important performance boost. In addition, we evaluated the energy efficiency.

Future work will be oriented to the design of efficient adaptive schemes. In addition, a testbed implementation will be considered to validate experimentally the conclusions drawn from the analytical and simulation models.

\section{Acknowledgements}

We would like to thank you the anonymous reviewers whose insightful and constructive comments made it possible to greatly improve the final version of this paper.

\section{References}

[1] 3GPP, TS 22.368, service Requirements for Machine-Type Communications, 2004.
[2] R. Cavallari, F. Martelli, R. Rosini, C. Buratti, R. Verdone, A survey on wireless body area networks: Technologies and design challenges, IEEE Communications Surveys \& Tutorials 16 (3) (2014) 1635-1657.

[3] N. Abramson, The ALOHA system: another alternative for computer communications, in: Proceedings of the November 17-19, 1970, fall joint computer conference, ACM, 1970, pp. $281-285$.

[4] L. G. Roberts, Extensions of packet communication technology to a hand held personal terminal, in: Proceedings of the May 16-18, 1972, spring joint computer conference, ACM, 1972, pp. 295-298.

[5] H. Okada, Y. Igarashi, Y. Nakanishi, Analysis and application of framed aloha channel in satellite packet switching networksfadra method, Electronics Communications of Japan 60 (1977) $72-80$.

[6] W. Szpankowski, Analysis and stability considerations in a reservation multiaccess system, IEEE Transactions on Communications 31 (5) (1983) 684-692.

[7] J. E. Wieselthier, A. Ephremides, L. A. Michaels, An exact analysis and performance evaluation of framed ALOHA with capture, IEEE Transactions on Communications 37 (2) (1989) $125-137$.

[8] K. Finkenzeller, RFID Handbook, Wiley Online Library, 2003.

[9] X. Xiong, K. Zheng, R. Xu, W. Xiang, P. Chatzimisios, Low power wide area machine-to-machine networks: key techniques and prototype, IEEE Communications Magazine 53 (9) (2015) 64-71.

[10] F. Vázquez-Gallego, J. Alonso-Zarate, L. Alonso, Reservation dynamic frame slotted-ALOHA for wireless M2M networks with energy harvesting, in: 2015 IEEE Int. Conf. on Communications (ICC), IEEE, 2015, pp. 5985-5991.

[11] W. Crowther, R. Rettberg, D. Walden, S. Ornstein, F. Heart, A system for broadcast communication: Reservation-ALOHA, in: Proc. 6th Hawaii Int. Conf. Syst. Sci, 1973, pp. 596-603.

[12] B. T. Doshi, H. Heffes, Overload performance of several processor queueing disciplines for the $M / M / 1$ queue, IEEE Transactions on Communications COM-34 (6) (1986) 538-546.

[13] V. Casares-Giner, J. Martinez-Bauset, Performance evaluation of framed slotted ALOHA with reservation packets for M2M networks, in: 5th Int. Conf. on Future Internet of Things and Cloud (FiCloud), IEEE, 2017, pp. 18-24.

[14] B. Van Houdt, C. Blondia, Robustness of Q-ary collision resolution algorithms in random access systems, Performance Evaluation 57 (3) (2004) 357-377.

[15] J.-C. Huang, T. Berger, Delay analysis of interval-searching contention resolution algorithms, IEEE Transactions on Information Theory 31 (2) (1985) 264-273.

[16] M. L. Molle, G. C. Polyzos, Conflict resolution algorithms and their performance analysis, Tech. rep., University of Toronto, CS93-300, Tech. Rep (1993).

[17] R. Rivest, Network control by Bayesian broadcast, IEEE Transactions on Information Theory 33 (3) (1987) 323-328.

[18] J. Capetanakis, Tree algorithms for packet broadcast channels, IEEE Transactions on Information Theory 25 (5) (1979) 505515.

[19] G. Polyzos, M. Molle, A. Venetsanopoulos, Performance analysis of finite nonhomogeneous population tree conflict resolution algorithms using constant size window access, IEEE Transactions on Communications 35 (11) (1987) 1124-1138. 
[20] M. Paterakis, P. Papantoni-Kazakos, A simple window random access algorithm with advantageous properties, IEEE Transactions on Information Theory 35 (5) (1989) 1124-1130.

[21] W. Szpankowski, An analysis of a contention resolution algorithm - another approach, Tech. rep., Department of Computer Science, Report Number: 85-551. Purdue University (1985).

[22] F. Schoute, Dynamic frame length aloha, IEEE Transactions on Communications 31 (4) (1983) 565-568.

[23] G. Choudhury, S. Rappaport, Diversity ALOHA-a random access scheme for satellite communications, IEEE Transactions on Communications 31 (3) (1983) 450-457.

[24] E. Casini, R. De Gaudenzi, O. D. R. Herrero, Contention resolution diversity slotted ALOHA (CRDSA): An enhanced random access schemefor satellite access packet networks, IEEE Transactions on Wireless Communications 6 (4) (2007) 1408-1419.

[25] G. Liva, Graph-based analysis and optimization of contention resolution diversity slotted ALOHA, IEEE Transactions on Communications 59 (2) (2011) 477-487.

[26] Y. Yu, G. B. Giannakis, High-throughput random access using successive interference cancellation in a tree algorithm, IEEE Transactions on Information Theory 53 (12) (2007) 4628-4639.

[27] E. Paolini, G. Liva, M. Chiani, Coded slotted aloha: A graphbased method for uncoordinated multiple access, IEEE Transactions on Information Theory 61 (12) (2015) 6815-6832.

[28] A. Hernandez, F. Vazquez-Gallego, L. Alonso, J. AlonsoZarate, Performance evaluation of frame slotted-aloha with intra-frame and inter-frame successive interference cancellation, in: Global Communications Conference (GLOBECOM), 2015 IEEE, IEEE, 2015, pp. 1-6.

[29] L. Ping, L. Liu, W. Leung, A simple approach to nearoptimal multiuser detection: interleave-division multiple-access, in: Wireless Communications and Networking, 2003. WCNC 2003. 2003 IEEE, Vol. 1, IEEE, 2003, pp. 391-396.

[30] Y. Hu, C. Xu, L. Ping, NOMA and IDMA in random access systems - invited paper, in: 2018 IEEE 87th Vehicular Technology Conference (VTC Spring), 2018, pp. 1-5.

[31] M. Eroz, L. Lee, Scrambled coded multiple access, in: Proceedings of the 74th IEEE Vehicular Technology Conference, VTC Fall 2011, 5-8 September 2011, San Francisco, CA, USA, 2011, pp. $1-5$.

[32] N. Becker, M. Eroz, S. Kay, L. Lee, Asynchronous scrambled coded multiple access (A-SCMA) - a new high efficiency random access method, in: 2016 IEEE 84th Vehicular Technology Conference (VTC-Fall), 2016, pp. 1-6.

[33] N. Becker, S. Kay, L.-n. Lee, M. Eroz, Spread asynchronous scrambled coded multiple access (SA-SCMA)-a new efficient random access method, in: Global Communications Conference (GLOBECOM), 2016 IEEE, IEEE, 2016, pp. 1-6.

[34] F. Babich, M. Comisso, Segmented framed slotted aloha (SFSA) with capture and interference cancellation, in: 2018 IEEE 87th Vehicular Technology Conference (VTC Spring), IEEE, 2018, pp. 1-5.

[35] R. De Gaudenzi, O. Del Rio Herrero, G. Gallinaro, S. Cioni, P.-D. Arapoglou, Random access schemes for satellite networks, from VSAT to M2M: a survey, International Journal of Satellite Communications and Networking 36 (1) (2018) 66-107.
[36] L. G. Roberts, Dynamic allocation of satellite capacity through packet reservation, in: Proceedings of the June 4-8, 1973, national computer conference and exposition, ACM, 1973, pp. 711-716.

[37] F. Babich, Analysis of frame-based reservation random access protocols for microcellular radio networks, IEEE Transactions on Vehicular Technology 46 (2) (1997) 408-421. doi: $10.1109 / 25.580780$

[38] S. S. Lam, Packet broadcast networks: A performance analysis of the R-ALOHA protocol, IEEE Transactions on Computers C-29 (7) (1980) 596-603.

[39] S. Tasaka, Stability and performance of the R-ALOHA packet broadcast system, IEEE Transactions on Computers C-32 (8) (1983) 717-726.

[40] F. Vázquez-Gallego, J. Alonso-Zarate, A. M. Mandalari, O. Briante, A. Molinaro, G. Ruggeri, Performance evaluation of reservation frame slotted-ALOHA for data collection M2M networks, in: 20th European Wireless Conference, VDE, 2014, pp. 1-6.

[41] X. Chen, C. Huang, X. Fan, D. Liu, P. Li, Ldmac: A propagation delay-aware mac scheme for long-distance uav networks, Computer Networks 144 (2018) 40-52.

[42] X. Jiang, D. H. Du, Ptmac: A prediction-based tdma mac protocol for reducing packet collisions in vanet., IEEE Trans. Vehicular Technology 65 (11) (2016) 9209-9223.

[43] A. Laya, C. Kalalas, F. Vazquez-Gallego, L. Alonso, J. AlonsoZarate, Goodbye, ALOHA!, IEEE Access 4 (2016) 2029-2044.

[44] V. Casares-Giner, V. Sempere-Payá, D. Todolí-Ferrandis, Framed ALOHA protocol with FIFO-blocking and LIFO-push out discipline., Network Protocols and Algorithms 6 (3) (2014) $82-102$.

[45] L. Tello-Oquendo, V. Pla, I. Leyva-Mayorga, J. MartinezBauset, V. Casares-Giner, L. Guijarro, Efficient random access channel evaluation and load estimation in LTE-A with massive MTC, IEEE Transactions on Vehicular Technology (in press).

[46] M. F. Neuts, Matrix-geometric solutions in stochastic models: an algorithmic approach, The Johns Hopkins University Press, 1981

[47] A. S. Alfa, Queueing Theory for Telecommunications: Discrete Time Modelling of a Single Node System, Springer, 2010.

[48] G. Bolch, S. Greiner, H. De Meer, K. S. Trivedi, Queueing networks and Markov chains: modeling and performance evaluation with computer science applications, John Wiley \& Sons, 2006.

[49] M. Zorzi, R. R. Rao, Capture and retransmission control in mobile radio, IEEE Journal on Selected Areas in Communications 12 (8) (1994) 1289-1298.

[50] R. Porat, S. Yong, IEEE P802.11 wireless LANs, TGah channel model, proposed text, Tech. rep., IEEE 802.11-11/0968r1 (2011).

[51] A. Hazmi, J. Rinne, M. Valkama, Feasibility study of IEEE 802.11ah radio technology for IoT and M2M use cases, in: IEEE GLOBECOM Workshops, 2012, pp. 1687-1692.

[52] J. Martinez-Bauset, V. Casares-Giner, C. Portillo, FSA-RDP with successive interference cancellation, Tech. rep., Instituto ITACA, Universitat Politècnica de València, Spain (2019). URL http://personales.upv.es/jmartine/TR19.pdf 